\title{
Review
}

Zile Li, Shaohua Yu and Guoxing Zheng*

\section{Advances in exploiting the degrees of freedom in nanostructured metasurface design: from 1 to 3 to more}

https://doi.org/10.1515/nanoph-2020-0127

Received February 17, 2020; revised March 22, 2020; accepted March 23, 2020

\begin{abstract}
The unusual electromagnetic responses of nanostructured metasurfaces endow them with an ability to manipulate the four fundamental properties (amplitude, phase, polarization, and frequency) of lightwave at the subwavelength scale. Based on this, in the past several years, a lot of innovative optical elements and devices, such as metagratings, metalens, metaholograms, printings, vortex beam generators, or even their combinations, have been proposed, which have greatly empowered the advanced research and applications of metasurfaces in many fields. Behind these achievements are scientists' continuous exploration of new physics and degrees of freedom in nanostructured metasurface design. This review will focus on the progress on the design of different nanostructured metasurfaces for lightwave manipulation, including by varying/fixing the dimensions and/ or orientations of isotropic/anisotropic nanostructures, which can therefore provide various functionalities for different applications. Exploiting the design degrees of freedom of optical metasurfaces provides great flexibility in the design of multifunctional and multiplexing devices, which can be applied in anticounterfeiting, information encoding and hiding, high-density optical storage, multichannel imaging and displays, sensing, optical communications, and many other related fields.
\end{abstract}

*Corresponding author: Guoxing Zheng, Electronic Information School, Wuhan University, Wuhan 430072, China, e-mail: gxzheng@whu.edu.cn. https://orcid.org/0000-00023226-4735

Zile Li: Electronic Information School, Wuhan University, Wuhan 430072, China; and Suzhou Institute of Wuhan University, Suzhou 215123, China

Shaohua Yu: NOEIC, State Key Laboratory of Optical Communication Technologies and Networks, Wuhan Research Institute of Posts \& Telecommunications, Wuhan 430074, China
Keywords: optical metasurface; geometric phase; holography; metalens; degrees of freedom; multifunctional device.

\section{Introduction}

Scientists have been exploring for hundreds of years how to control lightwave more precisely. However, limited by the principles of controlling lightwave with the classic Snell's law, the functionality of traditional refractive and reflective optical elements is simple, which makes it difficult to realize the miniaturization, array, and integration of optical elements with arbitrary wavefront transformation. Although continuous- or binary-relief diffractive optical elements can achieve the arbitrary control of lightwave to the greatest extent in principle, due to the limitations of available materials, phase steps, pixel resolution, etc., its deep-level application has encountered great technical obstacles, which can be expected to be very difficult to break in the near future. Therefore, academia and industry are looking forward to the innovation of lightwave manipulation based on new concepts, new principles, and new technologies.

In recent years, scientists have proposed periodic subwavelength structures carved on the surface of ordinary optical materials. As subwavelength structure arrays, named as metasurfaces later, have unusual electromagnetic properties, they can be employed to control the incident electromagnetic field at the subwavelength scale [1-11]. Metasurfaces can precisely control the incident wavefront and can readily achieve complex lightwave manipulation, such as correction of lens aberrations, three-dimensional (3D) holography, and so on. In addition, the geometry and orientation of each unit cell of a metasurface can be controlled independently, providing the design degrees of freedom for the lightwave control, as shown in Figure 1. Therefore, compared to conventional optical elements, metasurfaces can be

O Open Access. (c) 2020 Guoxing Zheng et al., published by De Gruyter. (cc) BY Public License. 

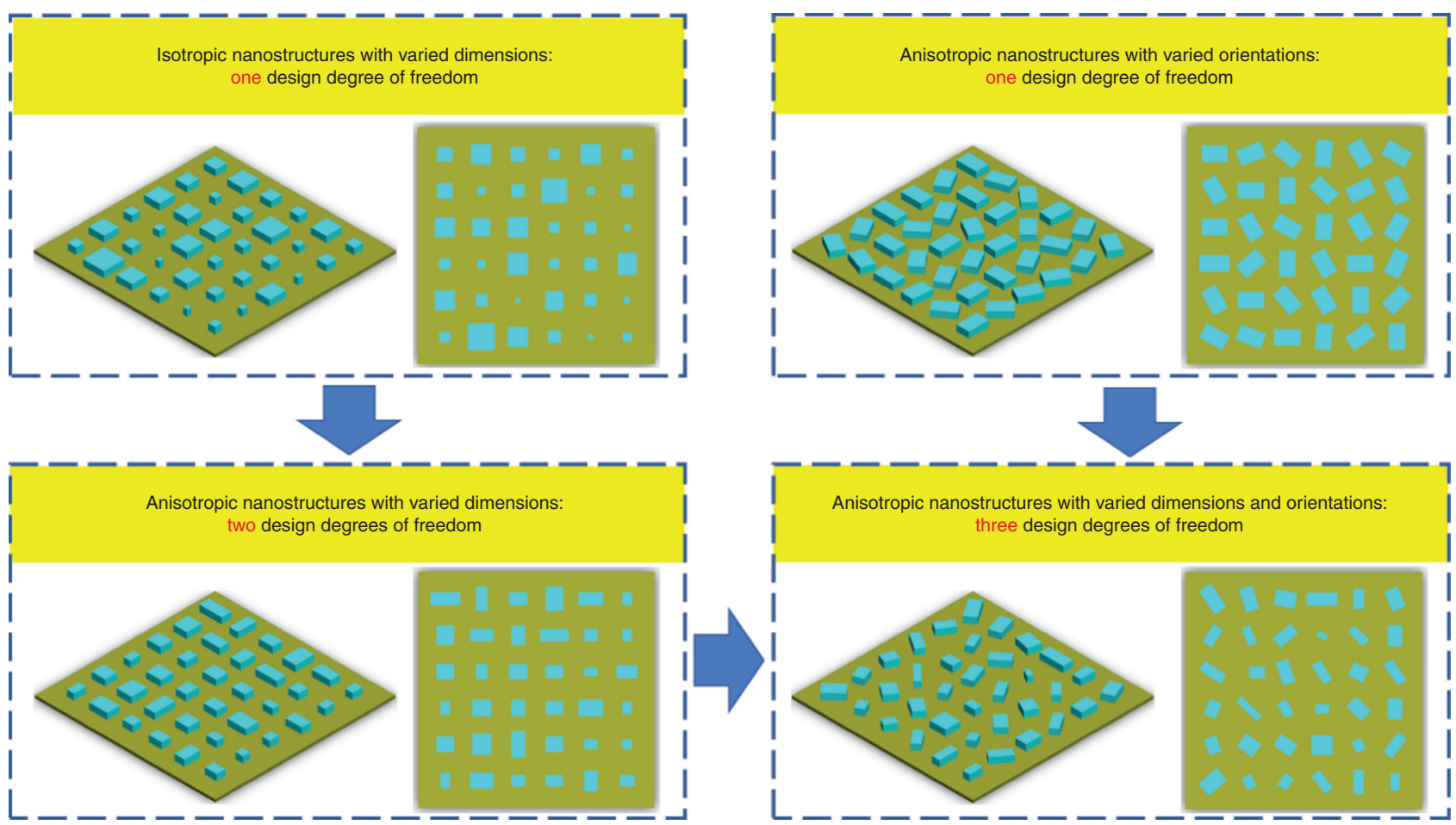

Figure 1: Schematic of the degrees of freedom in nanostructured metasurface design: from 1 to 3. More design degrees of freedom can be acquired with multilayered (stacked) and dynamic metasurfaces.

employed to realize more complex and integrated multifunctional devices, such as highly integrated achromatic lens, zoom lens, color holography, and polarization camera.

Considering that the more degrees of freedom the nanostructures have in the design, the more complex and richer functionalities can be realized, we focus on advances in exploiting the degrees of freedom in nanostructured metasurface design in this paper. Specifically, we start from the generalized law of refraction and reflection proposed by the Capasso research group (Section 2), introduce the metasurfaces with single functionality, review the development of nanostructured metasurfaces from isotropy (Section 3) to anisotropy (Section 4), focus on manipulating the phase, polarization, and intensity of lightwave by rotating the orientations of nanostructures (Section 5), and analyze the working principles of geometric metasurfaces and their applications. By changing the anisotropy of nanostructures in different cells of metasurfaces while the geometric phase remains unchanged, the more compact and multifunctional metasurfaces can be realized (Section 6). Finally, the future development of metasurfaces is prospected.

\section{V-shaped antennas and the generalized laws of refraction and reflection of lightwave}

In 2011, Yu et al. from Harvard University proposed the generalized law of refraction and reflection (Figure 2A) [12]. Different from the traditional Snell's law of refraction and reflection, the generalized law of refraction and reflection shows that the direction of output light is related not only to the direction of incident light but also to the phase gradient of the interface between two optical media. According to the continuous boundary conditions of electric field derived from Maxwell equations, the phase gradient of output light on the interface is the sum of the phase gradient of incident light on the interface and the phase gradient $\mathrm{d} \varphi(x) / \mathrm{d} x$ introduced by the interface itself [considering the one-dimensional (1D) situation]. Viewed from another perspective, the electric field of output light is equal to the electric field of incident light multiplied by the transmittance or reflectance coefficients of the interface, which can be expressed as $A(x) \cdot \exp [i . \varphi(x)]$. In the Fourier spatial frequency domain, the electric field of output light is equal to that of 

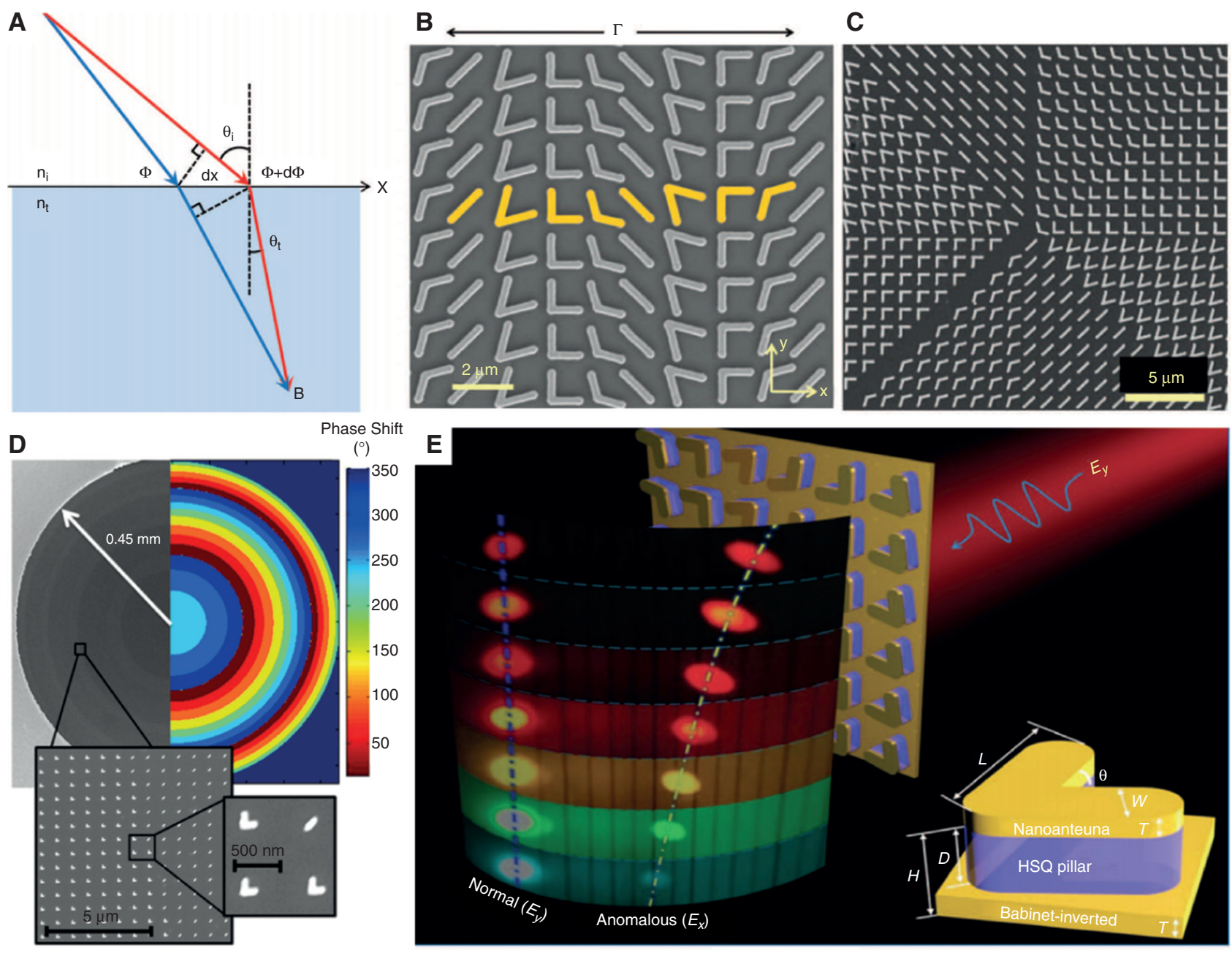

Phase Shift
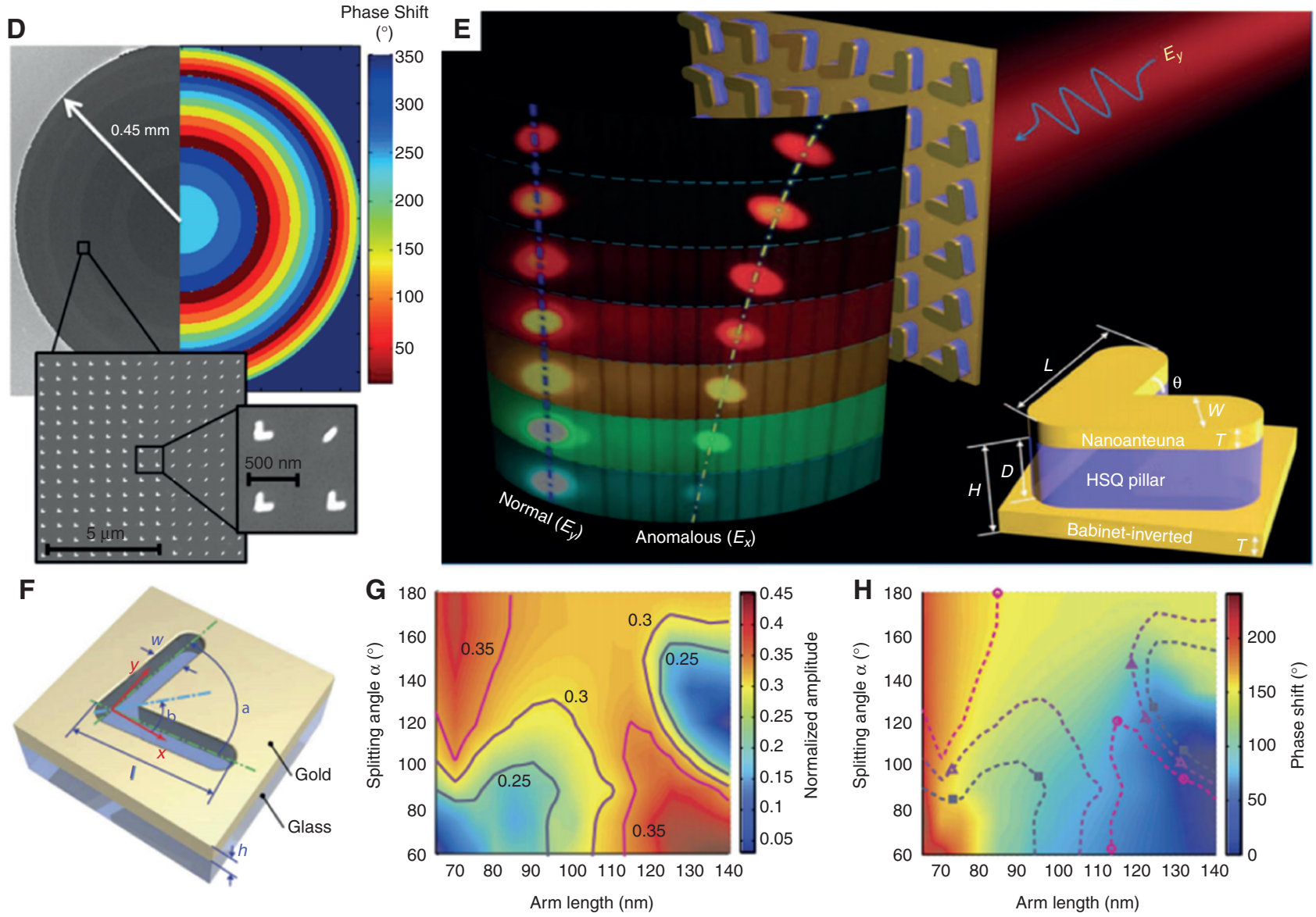

Figure 2: Generalized law of refraction and reflection and V-shaped antennas.

(A) Generalized law of refraction and reflection [12]. (B-D) Metagrating [12], spiral phase plate [12], and metalens [13] based on V-shaped antennas. (E) Metagrating based on dual-layer V-shaped antennas [14]. (F-H) Complex amplitude manipulation based on V-shaped antennas [15]. Reprint permission obtained from [12-15].

incident light convolved with $f\left(k_{x}\right)=F T\{A(x) \cdot \exp [i . \varphi(x)]\}$ at the interface, where $k_{x}$ is the spatial frequency caused by the phase gradient in the $x$-axis and FT denotes the operator of Fourier transformation. In the case of normal incidence, as the electric field of incident light is $\delta(0)$ at the interface, the electric field of output light is the same as $f\left(k_{x}\right)$. In the case of oblique incidence, the electric field of incident light is $\delta\left(k_{x i}\right)$ at the interface, where $k_{x i}=\sin \left(\theta_{i}\right) / \lambda$ and $\theta_{i}$ is the incident angle. In this case, the spatial frequency of output light $k_{x o}$ can be obtained by adding $k_{x i}$ and $k_{x}$ together. According to the relationship between the spatial frequency and the output angle, i.e. $k_{x o}=\sin \left(\theta_{o}\right) / \lambda$, the output angle $\theta_{o}$ can be obtained. Based on the above principle, the research group gave 
a design case and experimental samples of metasurfaces composed of V-shaped metallic nanostructures with different dimensions and shapes to produce phase gradient $\mathrm{d} \varphi(x) / \mathrm{d} x$ and used it to design a blazed grating (Figure 2B) and a vortex beam generator (Figure 2C). This work is the first to demonstrate that periodic subwavelength structures have quite different light manipulation characteristics from the traditional theory, which started an upsurge in the research on metasurfaces.

Based on this work, in 2012, Aieta et al. from the same research group used a group of V-shaped antennas that can produce different phase delays to design a lens with spherical aberration correction (Figure 2D) and an axicon mirror that can generate a diffraction-free Bessel beam at a wavelength of $1.55 \mu \mathrm{m}$ (the optical fiber communication window) [13]. Experimental verification was carried out, further proving the flexibility of the precise control of lightwave with metasurfaces. However, most metals have inherent loss in optical ranges, and it is difficult to achieve impedance matching with the surrounding optical medium (e.g. air), resulting in low transmittance of metallic nanostructures with variable shapes (the experimental efficiency is $\sim 1 \%$ ), which is difficult to meet the practical requirements.

To solve the efficiency problem of V-shaped metallic nanostructures, Qin et al. proposed a dual-layer nanostructure by coupling the nanoantenna with its complementary Babinet-inverted copy (Figure 2E) [14]. A blazed grating based on metasurfaces was designed with the nanostructures and verified by experiments, with an improved efficiency of $17 \%$.

In addition to phase control, $\mathrm{Ni}$ et al. realized the complex amplitude control of lightwave by flexibly designing the arm length of V-shaped metallic holes and the angle between two nanoarms (Figure $2 \mathrm{~F}-\mathrm{H}$ ) [15]. Based on this structure, an ultrathin (30 $\mathrm{nm}, \sim \lambda / 23)$ metasurface hologram with high resolution and low noise was obtained. The proposed scheme extends the functionality of metasurfaces and provides a new path for lightwave manipulation.

\section{Isotropic nanostructures with varied dimensions as a design degree of freedom}

In addition to V-shaped nanostructures, inspired by the wavefront control approach with traditional optical components, researchers have also studied other methods for generating phase gradients.
In the traditional design of optical components, the phase of incident light is manipulated through optical path difference to adjust the wavefront of transmitted or reflected light. The relationship between the phase delay of the optical wavefront $\varphi$ and the optical path difference $L$ is as follows:

$$
\varphi=2 \pi L / \lambda,
$$

where $\lambda$ is the wavelength of incident light. The optical path difference $L$ is determined by the refractive index $n$ and the light propagation distance $s$, i.e. $L=n$.s. According to Eq. (1), there are two ways to change the optical path difference $L$ in the traditional optical element design. One method is to adjust the propagation distance $s$ of lightwave in an optical material, while the refractive index $n$ remains unchanged, such as the traditional spherical lens and binary optical elements $[16,17]$. Another method is to change the profiles of the refractive index $n$ of the material, while the propagation distance $s$ remains unchanged, such as gradient index (GRIN) lens [18, 19]. Phase manipulations by both ways are independent of the polarization state of incident light. However, the traditional spherical lens and GRIN lens can only be employed for simple wavefront control (e.g. conversion of spherical wave to plane wave or vice versa); therefore, the design flexibility is limited. For binary optical elements, the phase manipulation is conducted by etching different depths cell-by-cell; however, the phase steps and the manufacturing complexity are contradictory with each other.

In planar optical elements composed of subwavelength nanostructures, the "equivalent refractive index" $n$ can be modulated flexibly by changing the geometric sizes of nanostructures. When the nanostructure is isotropic, the light control of a planar optical element is independent of the polarization state of incident light. For example, in 2010, Paul et al. proposed a planar lens with gradient refractive index using metallic annular slots (Figure 3A) [20]. By changing the groove radii, the resonance characteristics of electromagnetic field can be controlled, so that the equivalent refractive index will be controlled. At an operating frequency of $1.2 \mathrm{THz}$, the difference of the equivalent refractive index is as high as 1.5.

Compared to complex metallic nanostructures, the use of post-nanostructures is a simpler scheme to achieve the control of the equivalent refractive index. By changing the side lengths of the metallic nanoscale pillars (Figure 3B), Verslegers et al. realized the plane focusing lens at a wavelength of $632.8 \mathrm{~nm}$ [21]. Zhang et al. employed a subwavelength dielectric post-array to realize a broadband focusing lens working in the 


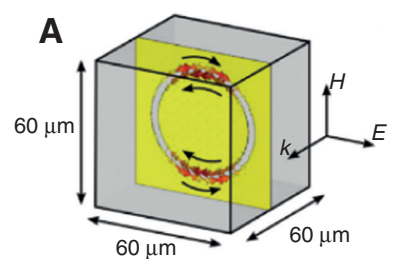

B
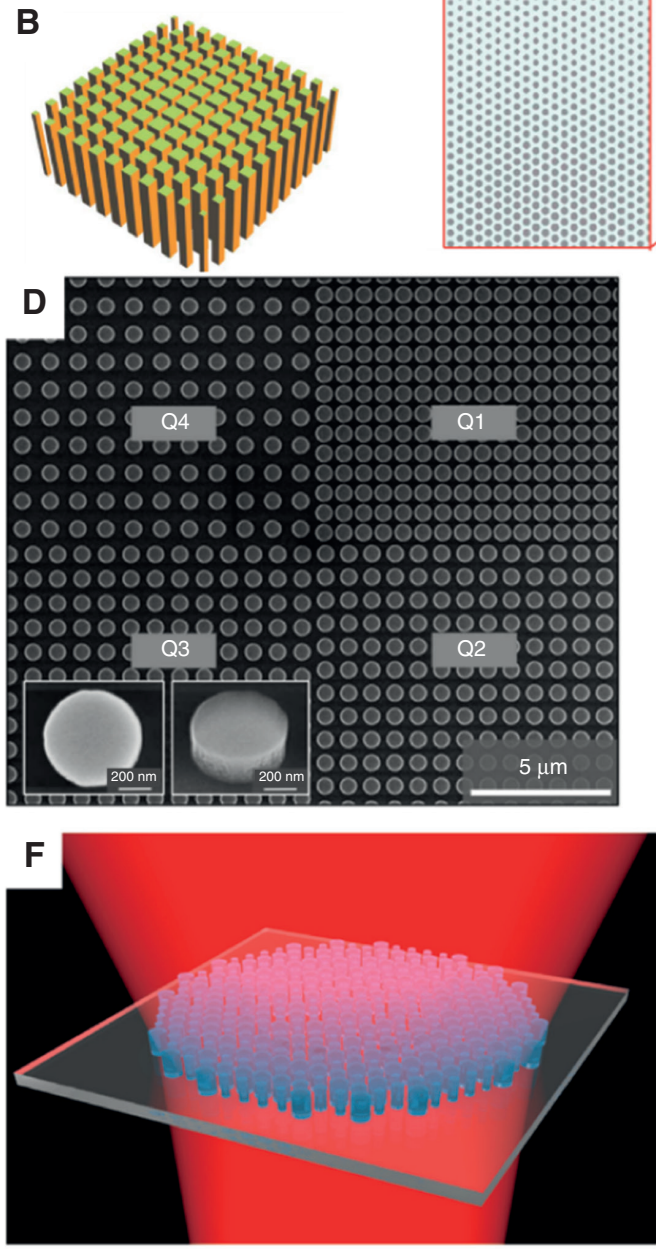

C

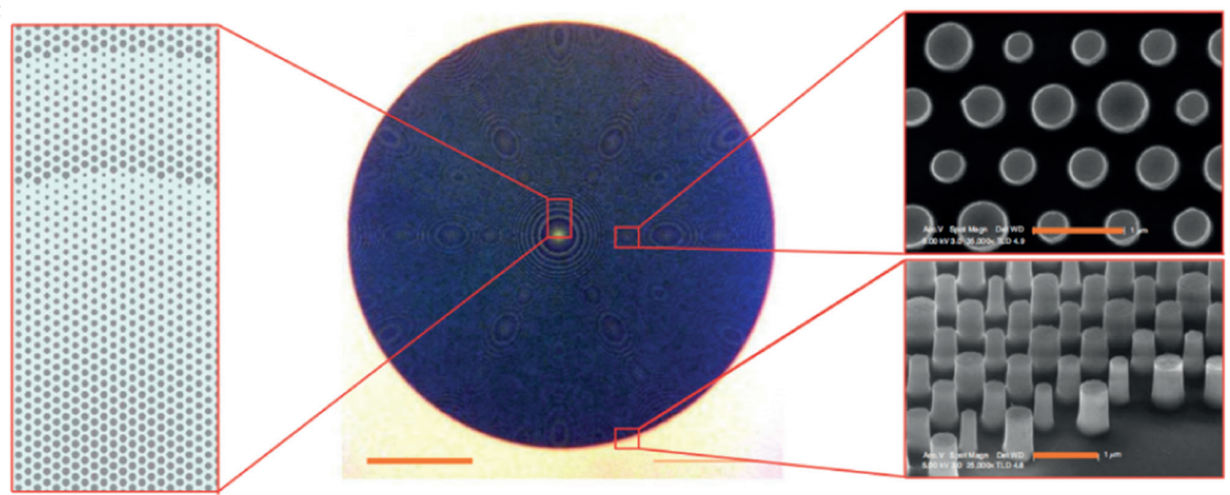

E
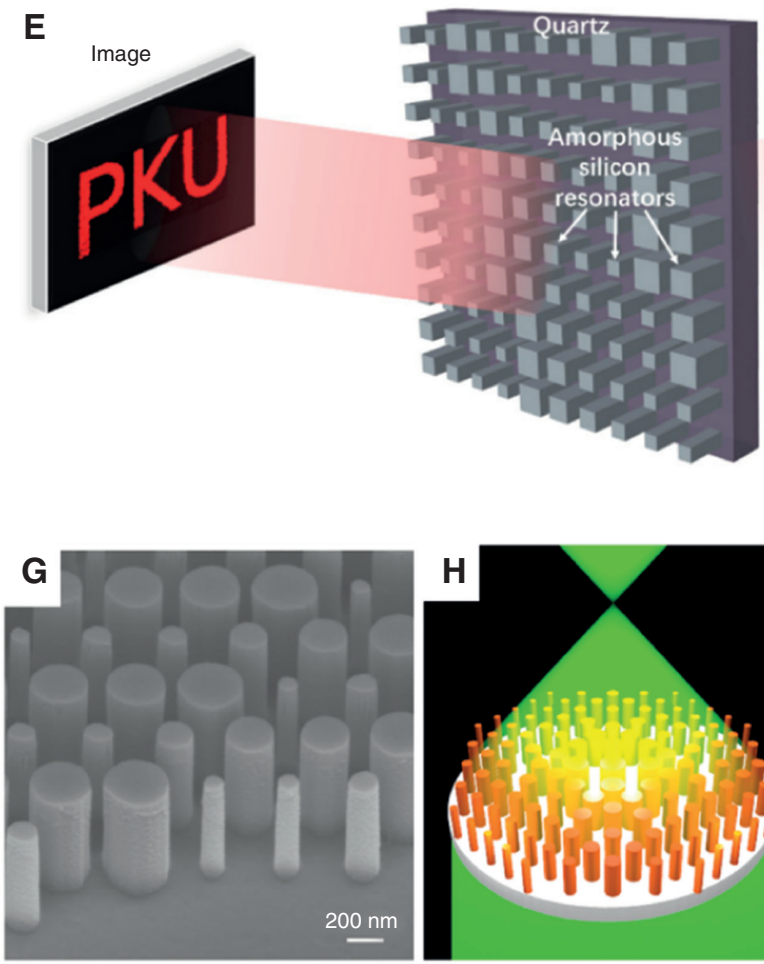

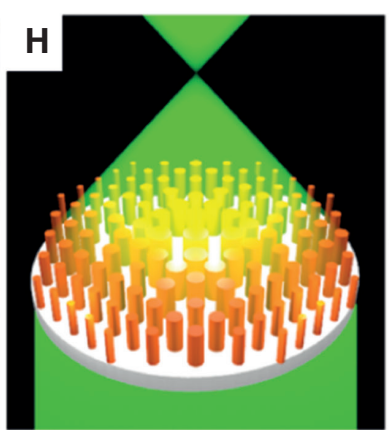

Figure 3: Isotropic nanostructured metasurfaces.

(A) Metallic annular slots [20]. (B) Metalens based on metallic nanoscale pillars [21]. (C) Metalens based on silicon nanoposts with varied radii [22]. (D) Spiral phase plate based on silicon nanoposts with equal radius but varied spacing between nanoposts [23]. (E) Visible light holography based on amorphous silicon nanoresonators [24]. ( $F$ and G) Metalens based on silicon nitride nanoposts and 3D SEM image in partial view [25]. (H-J) Metalens based on $\mathrm{TiO}_{2}$ nanoposts [26]. Reprint permission obtained from [20-26].

millimeter-wave range [27]. However, due to the limitation of nanofabrication technology, most early research works were based on numerical simulations without experimental verification, especially in the short wavelength band.

With the development of advanced nanofabrication, Arbabi et al. designed and fabricated a metalens (Figure 3C) with large numerical aperture (NA) and high efficiency at a working wavelength of $1.55 \mu \mathrm{m}$ [22]. The metalens is composed of silicon nanoposts sitting on a transparent substrate. The height of the nanopost is fixed at $940 \mathrm{~nm}$, and by varying the radii of the nanoposts, the phase difference can cover $0-2 \pi$. Thus, the phase of incident light can be manipulated point-by-point, which solves the problem that traditional large NA lens requires a complex surface shape to correct the spherical aberration. At the same time, due to the low loss of silicon in the optical communication band, the transmission of nanoposts with different radii can be kept at a high level through reasonable design. The measured diffraction focusing efficiency of the metalens is as high as $82 \%$, and the full-width at half-maximum spot is $0.57 \lambda$. 
In addition to varying the radii of nanoposts, Chong et al. designed a cylindrical nanostructure based on Mie resonance. By varying the spacing between the nanoposts, the phase difference coverage of $0-2 \pi$ was attained when the working wavelength was $1.477 \mu \mathrm{m}$. The metasurface to generate a vortex beam with Gaussian wave incidence was designed and verified by experiments (Figure 3D) [23]. More importantly, the introduction of Mie resonance greatly reduces the height of the nanoposts $(243 \mathrm{~nm}$ in their design), thus reducing the difficulty of silicon etching. At the same time, the strongly localized electric and magnetic fields in the silicon nanoparticles can suppress the backscattering, that is, the transmittance of nanostructures is nearly $100 \%$.

As a kind of material commonly used in semiconductor technology, silicon is also employed in the metasurface design in the visible band. Because silicon has a high refractive index, it is easy to generate a large difference of the equivalent refractive index. In 2016, Li et al. designed six square nanoresonators with different side lengths using amorphous silicon. They designed and fabricated six-step phase-only holograms (Figure 3E) and obtained reconstructed holographic images with high fidelity in the wide band range of 473-700 $\mathrm{nm}$ [24]. Due to the loss of amorphous silicon in visible light, the measured efficiency of the metahologram is $31 \%$ when the working wavelength is $633 \mathrm{~nm}$.

Low-loss materials are generally not high refractive index materials in the visible range. Therefore, by varying the dimensions of nanostructures, the adjustable range of the equivalent refractive index is limited. To cover $0-2 \pi$ phase shift range, the height of the nanostructure has to be increased. However, with the increase of nanostructure height, resonance is more likely to occur, which significantly reduces the transmission and leads to the unwanted phase abruption. Reducing the cell size of the nanostructures can solve this problem. However, fabricating nanostructures with high aspect ratio has to face manufacturing difficulty. By reasonably selecting the nanostructure parameters to avoid the resonance peak, Zhan et al. designed and fabricated a silicon nitride metalens with a NA of 0.75 , transmission efficiency of $90 \%$, and focusing efficiency of $40 \%(\lambda=633 \mathrm{~nm})$ [28]. This scheme provides an idea for the design and fabrication of low-loss metasurfaces. In 2018, Fan et al. also designed and fabricated a metalens (Figure $3 \mathrm{~F}$ and $\mathrm{G}$ ) with the NA up to 0.98 using silicon nitride and carried out imaging experiments [25].

In contrast, the development of advanced manufacturing technology solves the problem of fabricating nanostructures with high aspect ratio. Khorasaninejad et al. fabricated titanium dioxide $\left(\mathrm{TiO}_{2}\right)$ nanostructures with a maximum aspect ratio of 6 using atomic layer deposition (ALD) technology and fabricated high-efficiency and large NA metalenses (Figure $3 \mathrm{H}-\mathrm{J}$ ) at red $(\lambda=633 \mathrm{~nm})$, green $(\lambda=532 \mathrm{~nm})$, and blue $(\lambda=405 \mathrm{~nm})$ bands, respectively, with a maximum NA of 0.85 , a maximum efficiency of $90 \%$, and a minimum focus spot of $0.64 \lambda$. The imaging experiment was carried out with the fabricated metalens, and the high-resolution image was observed [26].

High-performance isotropic metasurfaces are widely used in beam collimation, focusing, and imaging, as they are insensitive to the polarization of incident light. Arbabi et al. used a metalens with a NA of 0.86 and a transmission efficiency of $79 \%$ to collimate the midinfrared (IR) quantum cascade lasers (Figure 4A) with a large divergence angle of $55^{\circ}$ [29]. After collimation, the divergence angle is suppressed to be only $0.36^{\circ}$, and the beam quality factor is $\mathrm{M}^{2}=1.02$. In 2016, using two dielectric metasurfaces sitting on both sides of a glass substrate (Figure 4B), they realized a fisheye lens with a large F-number of 0.9 and a wide field-of-view (FOV) angle of $60^{\circ} \times 60^{\circ}$ [30]. With corrected monochromatic aberrations, the double-layered metasurface has great potential in the design of high-performance, low-loss, and lightweight passive optical elements. In 2017, they used double-layered metasurfaces to realize planestructured retroreflectors (Figure 4C) in the near-IR band, with high performance in the incident angle range of $\pm 60^{\circ}$ [31]. In 2018, Colburn et al. fabricated a metasurface with a diameter of $1 \mathrm{~cm}$ using high-throughput stepper photolithography [32] and achieved a largescale continuous-zoom metalens by changing the lateral displacement of two metasurface-based phase plates (Figure 4D). In the same year, Melissa J. Suter and the Capasso research group jointly developed a high-resolution endoscope using metasurfaces (Figure 4E-H) [33]. Their approach solves the constraints between transverse resolution and imaging depth in endoscope and realizes the in vivo detection of lung specimens and the upper respiratory tract of sheep. The proposed metasurface endoscope with high resolution and large imaging depth is expected to improve the clinical applicability of optical endoscope.

In addition to effectively correcting monochromatic aberrations with isotropic metasurfaces, Shrestha et al. designed nanocylinders with complex cross-section shapes (Figure 4I-K), which have unique dispersion characteristics [34]. Using optimized nanostructures, they designed and fabricated a metalens that can correct the chromatic aberration in a broad range of 
A

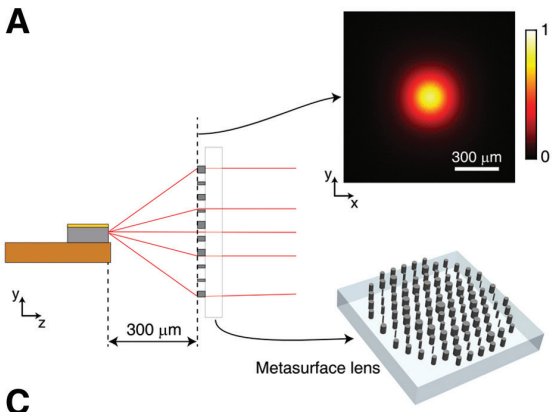

C

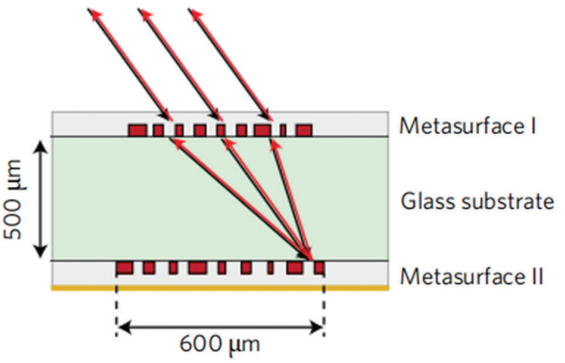

B

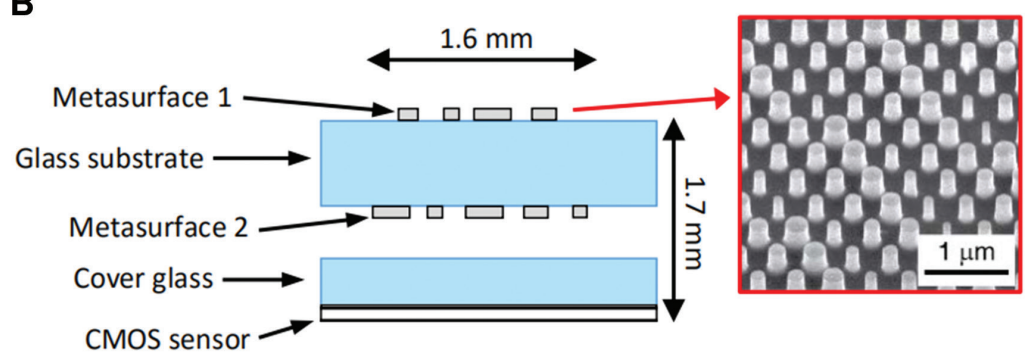

D

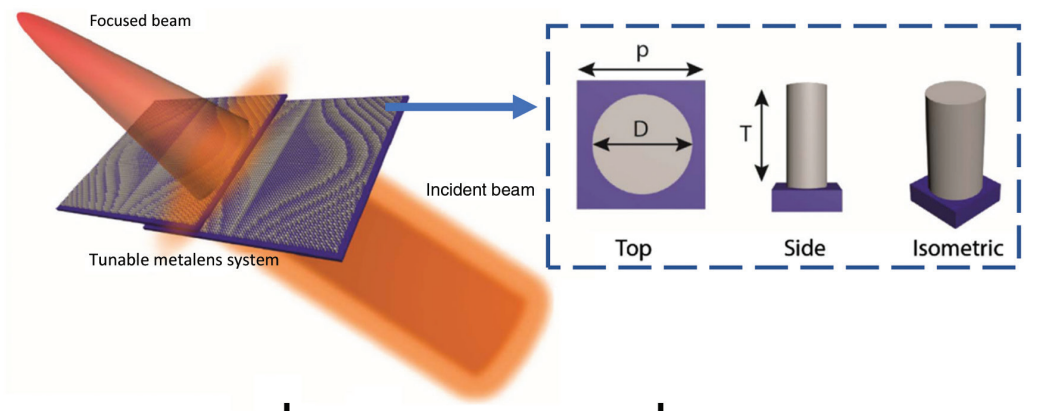

E

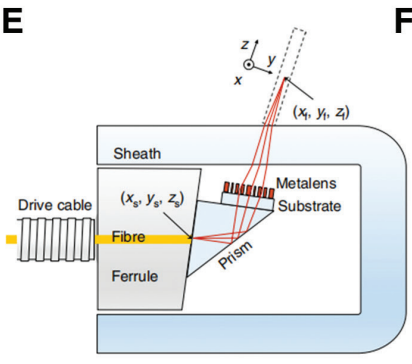

G

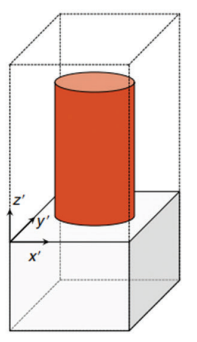

$\mathbf{F}$

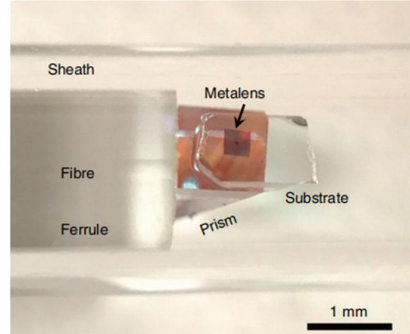

I Generation 1B
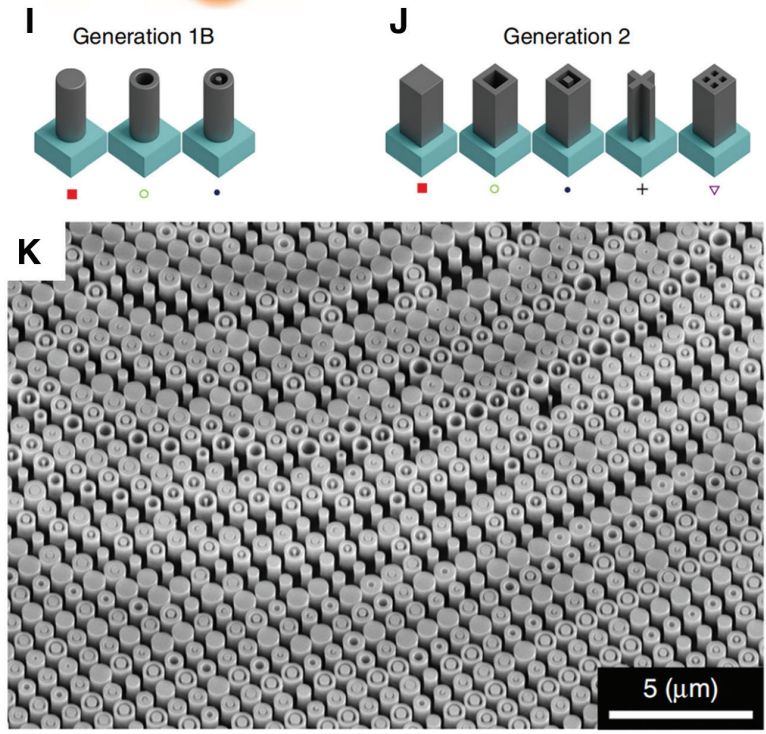

Figure 4: Applications of isotropic nanostructured metasurfaces with varied dimensions.

(A) Beam collimator for mid-IR quantum cascade lasers [29]. (B) Fisheye lens with large F-number and wide FOV [30]. (C) Plane-structured retroreflector based on double-layered metasurfaces [31]. (D) Large-scale continuous-zoom metalens by changing the lateral displacement of two phase-modulated metasurfaces [32]. (E-H) Metasurfaces for endoscope and their 3D SEM image [33]. (I-K) Achromatic metalens based on nanocylinders with complex cross-sections and their 3D SEM image [34]. Reprint permission obtained from [29-34].

$1200-1650 \mathrm{~nm}$. The focusing efficiency of the metalens is $50 \%$, and it is insensitive to the polarization state of incident light.

Isotropic nanostructures can also be used to realize amplitude modulation to encode the desired information. For example, two-step amplitude modulation can be realized using photon sieves. Based on this principle, Huang et al. designed and fabricated metahologram and superfocusing lens that are polarization independent [35]. They further proposed the design of ultrabroadband and large angle-of-view holograms based on photon sieves [36]. In 2019, with photon sieves, Xu et al. realized two amplitude holograms that are related to each other [37]. In terms of nanoprintings, amplitude manipulation can enable grayscale image display, and with delicate design, color printings can be achieved when light beams with different wavelengths illuminate on the metasurface. Tan et al. [38] and Dai et al. [39] demonstrated the realization of nanoprinting by controlling the dimensions of isotropic nanostructures and the spacing among them. 


\section{Anisotropic nanostructures with varied dimensions as one or two design degrees of freedom}

Due to the symmetry of nanostructures, the adjustable geometric parameters of isotropic nanostructure are limited. Although the number of phase steps is unlimited in principle, the increment of geometric sizes has to be small if one requires a large number of phase steps, which then burdens the nanofabrication process. In the design of metagratings, there is a simple approach to solve the fabrication problem with trapezoidal nanoantennas (Figure 5A). Using this trapezoidal nanostructure, $\mathrm{Li}$ et al. realized the abnormal reflection in a wide visible range (Figure 5B) [40] and even realized that incident light beams with different wavelengths were reflected toward the opposite direction (Figure 5C) [41]. Gao et al. designed a metallic metagrating with a wide band of $400-700 \mathrm{~nm}$ [49]. Yang et al. designed a dielectric metagrating with a theoretical transmission efficiency of $88 \%$ at an operating wavelength of $751 \mathrm{~nm}$ [42]. However, trapezoid nanostructures are only suitable for optical elements that require continuous and linear phase modulation, and it is difficult to realize elements that can perform complex light manipulation such as holograms and vortex beam generators.

By adjusting the dimensions of two orthogonal directions in a metasurface plane, the design flexibility of light manipulation can be effectively increased. In 2012, Sun et al. used cuboid metallic nanostructures with different dimensions to manipulate the phase of the reflected light and used a metallic reflector to further improve the reflectivity (Figure 5D) [43]. The measured diffraction efficiency of the metagrating is $80 \%$ in a wide band $(750-900 \mathrm{~nm})$. In 2013, Pors et al. also designed a metasurface with eightstep phase modulation by employing cuboid metallic nanostructures with different dimensions and realized the functionality of concave mirror [50].

Different from the metallic nanostructures aforementioned, Shalaev et al. proposed an ultracompact dielectric metasurface operating in transmission mode (the working wavelength $\lambda=1.55 \mu \mathrm{m}$ and the nanostructure height $h=270 \mathrm{~nm}$ ), as shown in Figure 5E [44]. They introduced Mie resonance into anisotropic nanostructures and analyzed the influence of Mie resonance on the transmittance. Mie resonance includes magnetic and electric resonance modes. The magnetic resonance mode comes from the excitation of cyclic electric displacement current in a nanostructure, which makes the enhanced magnetic field localized in the center of the nanostructure. In contrast, when electric resonance occurs, the electric field in the center of the nanostructure is enhanced, whereas the magnetic field is vortex-like. When the frequency of electric resonance is equal to that of magnetic resonance, the nanostructures have unusual high transmittance. When only electric resonance or magnetic resonance occurs, the resonance band is accompanied by a strongly reflective characteristic. High-Q measurement can be designed using collective behavior of Mie resonances. Based on this, Andreas et al. published their research results of measuring molecular absorption spectrum with metasurfaces in 2018 (Figure 5F) [45]. A metasurface with resonant nanostructures is designed and its reflection peak has a narrowband characteristic. By varying the dimensions of the nanostructures, the peak wavelength of the reflected light can be selected. By arranging varied dimensions of nanostructures, the molecular absorption spectrum can be measured with high sensitivity in the mid-IR spectral domain. In 2019, using the similar principle, Yesilkoy et al. developed an ultrasensitive and label-free analytical platform for biosensing by combining dielectric metasurfaces with hyperspectral imaging [51].

Anisotropic nanostructures with varied dimensions can be employed to not only manipulate the phase of lightwave but also realize polarization conversion. For example, the nanostructure equivalent to a quarter-wave plate can realize the conversion between linearly polarized (LP) light and circularly polarized (CP) light [52], and the nanostructure equivalent to a half-wave plate can realize the rotation of polarization direction of incident LP light [46-48, 53, 54]. In 2014, Yang et al. designed a set of reflective half-wave plate nanostructures [46] with different phase delays along the fast axis by combining dielectric materials with metallic reflector (Figure 5G-I). They made use of these nanostructures to form metasurfaces to realize blazed gratings and vortex beam generators. When the included angle between the polarization direction of incident light and the orientation of the nanostructure is $45^{\circ}$, the metasurface can not only manipulate the phase of incident light but also rotate the polarization direction of incident light by $90^{\circ}$. The measured reflectivity of the cross-polarized light is more than $97 \%$ in a broad band of 1420-1620 nm. Using the above principle, other phase-only elements with polarization conversion can also be realized. Zhong et al. designed an active zoom metalens with the focal length adjustable within 10\% by combining it with liquid crystals [53]. Ding et al. realized reflective broadband polarization conversion of incident lightwave using metallic nanostructures [54]. In terms of the transmission mode, Wang et al. used a group of L-type nanostructures to build a metasurface (Figure $5 \mathrm{~J}$ and $\mathrm{K}$ ), 



Figure 5: Anisotropic nanostructures with varied dimensions.

(A-C) Trapezoidal nanostructures [40-42]. (D and E) Cuboid nanostructures [43, 44]. (F) Spectrometer composed of high-Q nanostructures enabled with Mie resonance [45]. (G-I) Cuboid nanostructures with both phase control and polarization conversion functionalities in the IR band and the simulated cross-polarized reflectivity and phase delays [46]. ( $\mathrm{J}$ and $\mathrm{K}$ ) L-shaped nanostructures with both phase control and polarization conversion functionalities and the simulation results [47]. ( $L$ and $M$ ) Nanofins with phase control and polarization conversion functionalities and the simulation results in the THz band [48]. Reprint permission obtained from [40-48].

which can generate multiple focal spots [47]. Wang et al. designed nanofins with varied dimensions and realized that holographic patterns varied with different diffraction distances in the terahertz band (Figure 5L and M) [48].

By further optimizing the dimensions of anisotropic nanostructures, the phase of orthogonal polarized incident light can be controlled independently. In 2018, Deng et al. designed a polarization-sensitive metasurface that can independently manipulate the phase delays in two orthogonal polarization directions [55]. By assigning different dimensions along the long and short axes of the dielectric nanobrick, respectively, the phase delays are different in the two orthogonal directions, which can be employed to build two independent phase-only 
holograms (Figure 6A). Martins et al. experimentally demonstrated the feasibility of polarization-controlled 3D holography using crystalline silicon in the visible light band [61]. Xie et al. designed a plasmonic nanoslit array with continuous phase modulation and 10-level amplitude control in two orthogonal polarization directions
[62]. Fu et al. designed a dual FOV step-zoom metalens that is composed of dielectric metasurfaces on both sides of a transparent substrate. When two orthogonal LP light beams are incident, respectively, the metalens has two different focal lengths [56]. At the same time, the back focal plane of the metalens remains unchanged in two
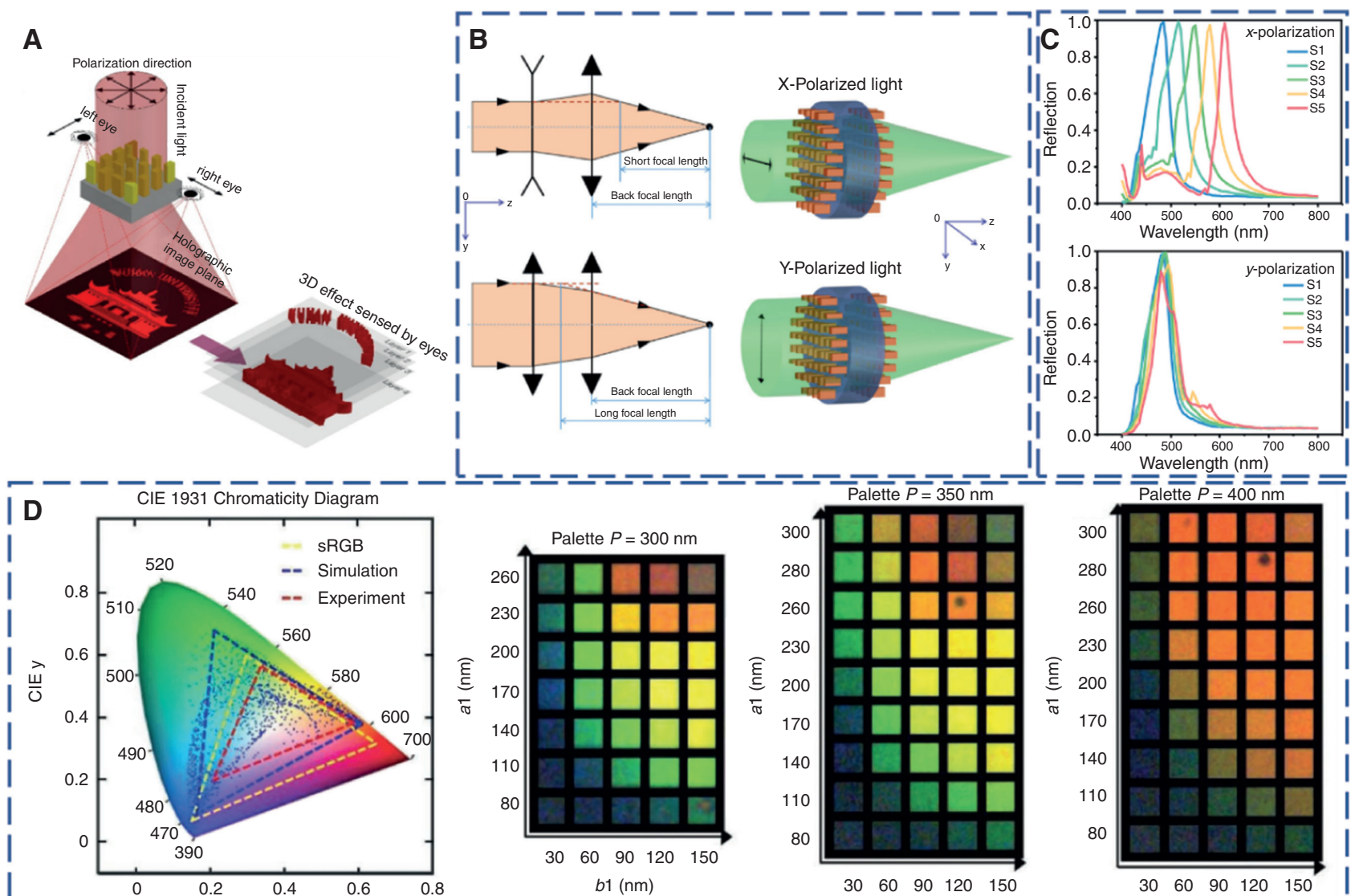

$30 \quad 60 \quad 90 \quad 120 \quad 150$

$b 1(\mathrm{~nm})$
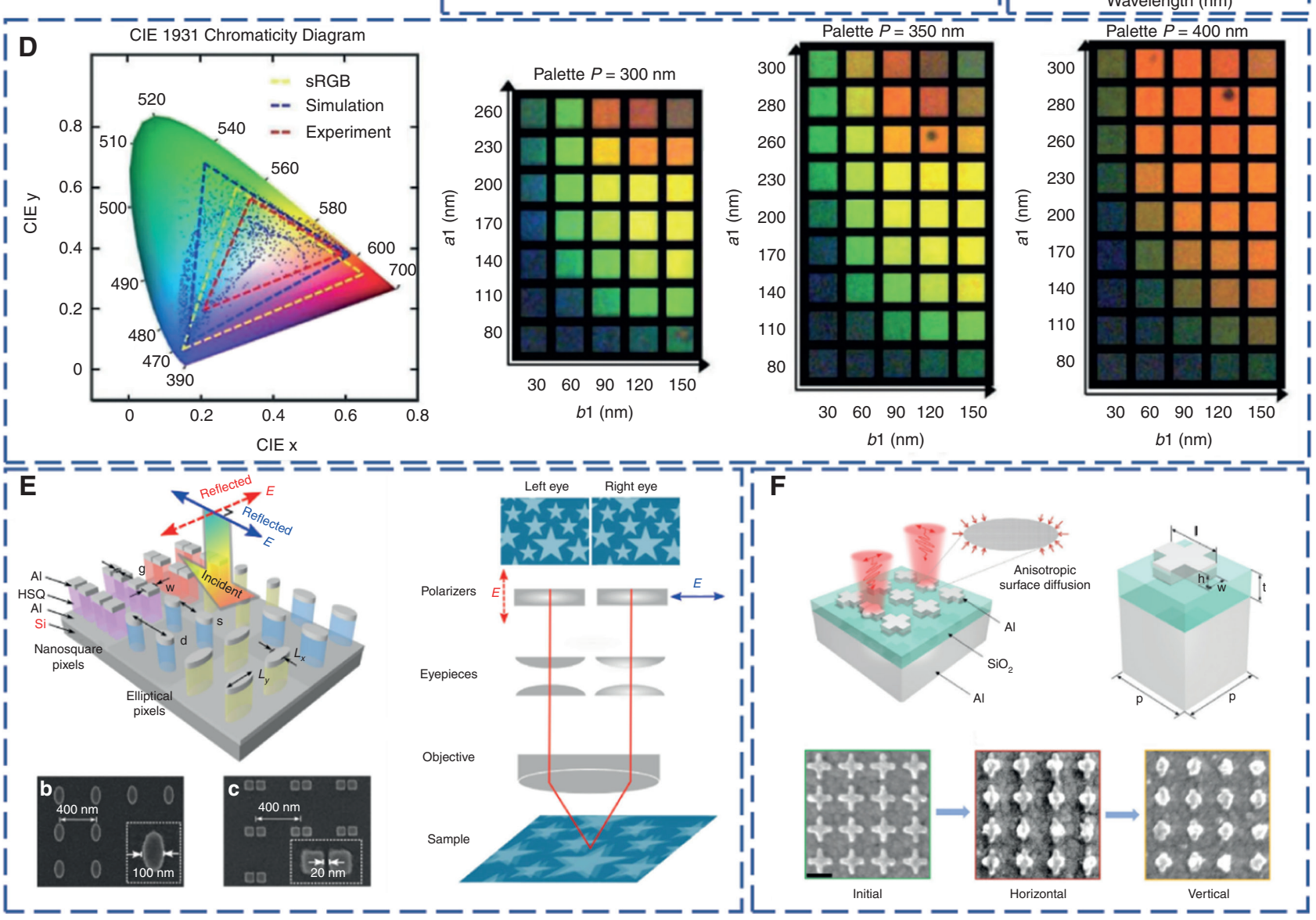

Figure 6: Anisotropic nanostructures with independent control of the phase and color in the orthogonal polarization directions. (A) 3D holographic display [55]. (B) Dual step-zoom metalens [56]. (C) Independent control of the spectral response in the orthogonal polarization directions [57]. (D) Wide-band full-color display [58]. (E) 3D nanoprintings for color image display [59]. (F) Metasurface fabrication using in situ anisotropic thermographic laser printing technology [60]. Reprint permission obtained from [55-60]. 
modes (Figure 6B), which brings great convenience to practical application.

By changing the dimensions of anisotropic nanostructures, one can not only manipulate the phase of incident light but also control the color by manipulating the transmitted and reflected spectra. For example, by varying the dimensions of dielectric nanostructures, the peak reflective wavelength of the metasurfaces shifts from $<500$ to $>600 \mathrm{~nm}$ when the $x$-axis LP light is incident [57]. As a result, the observed color changes correspondingly from blue to red. When LP light in the $y$-axis direction is incident, the reflected peak wavelength remains almost unchanged (Figure 6C), and the observed color is correspondingly unchanged. Jang et al. realized wide color-gamut and full-color display using nanostructures with different dimensions (Figure 6D) [58]. Goh et al. encoded two colorful images corresponding to the left and right eye views of human beings into a single metasurface and realized 3D color nanoprinting (Figure 6E) [59]. In 2019, Zhang et al. designed a multifunctional metasurface using in situ anisotropic thermographic laser printing technology (Figure 6F) [60]. When a femtosecond laser is used to irradiate cross-shaped aluminum nanostructures, the shape of the aluminum nanostructure will deform due to the thermal effect. Specifically, the varied lengths of two mutually perpendicular arms of the nanostructure will change the reflection spectrum characteristics when incident LP light is parallel to the polarization directions of the arms. Therefore, laser printing can control the color of each pixel on the metasurface, which provides a new scheme for both information multiplexing and metasurface fabrication.

\section{Anisotropic nanostructures with orientation control as a design degree of freedom}

There are still some shortcomings in the design of metasurfaces by varying the dimensions of nanostructures. First, for most optical elements, such as gratings and lenses in practical applications, it is appreciated to control the phase of incident lightwave rather than amplitude. However, the dimension differences of nanostructures can result in the change of the equivalent impedance. Therefore, the amplitude of incident light is still modulated with varied nanostructure dimensions. Second, because of the limitation of nanofabrication accuracy, the number of the phase modulation steps is limited. At last, the fabrication errors will also affect the accuracy of phase manipulation.

In recent years, geometric phase-based metasurfaces (GEMS) have been a wide concern due to their simple and robust phase control characteristic. The effect of GEMS on the phase and amplitude of incident light can be explained by Pancharatnam-Berry phase (P-B phase), which comes from the analysis and description of the additional phase of spin motion in the wave function of quantum spin state. The geometric phase is only related to the orientation of a nanostructure and independent of the nanostructure dimension and lightwave frequency. Furthermore, it is a phase-only method for phase manipulation, as each nanostructure in a GEMS is equal in dimensions, which effectively solves the shortcomings of metasurfaces with size-varied nanostructures. In fabrication, GEMS only need a twostep nano-optical process, so it has great commercial application prospects.

\subsection{P-B phase}

A Jones matrix of an optical element can be expressed as

$$
G=\left[\begin{array}{ll}
A & B \\
C & D
\end{array}\right]
$$

If we rotate the optical element with an angle $\alpha$ and

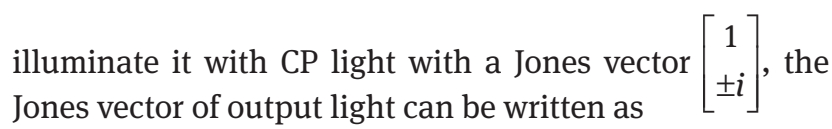

$$
\begin{aligned}
E_{\text {out }} & =\left[\begin{array}{cc}
\cos \alpha & -\sin \alpha \\
\sin \alpha & \cos \alpha
\end{array}\right]\left[\begin{array}{cc}
A & B \\
C & D
\end{array}\right]\left[\begin{array}{cc}
\cos \alpha & \sin \alpha \\
-\sin \alpha & \cos \alpha
\end{array}\right]\left[\begin{array}{c}
1 \\
\pm i
\end{array}\right] \\
& =p\left[\begin{array}{c}
1 \\
\pm i
\end{array}\right]+q e^{ \pm i 2 \alpha}\left[\begin{array}{c}
1 \\
\mp i
\end{array}\right]
\end{aligned}
$$

where $p$ and $q$ are expressed as

$$
p=\frac{A+D+(B-C)}{2} \text { and } q=\frac{A-D+(B+C)}{2} .
$$

It can be seen from the above formulas that the output field of CP light passing through the optical element is divided into two parts, i.e. CP light with the same and opposite handedness as incident light, respectively. The moduli of $p$ and $q$ represent the amplitudes of the two parts of CP light, respectively. It shows that only when $A=D$ and $B=-C$ (in this case, the matrix $G$ is an antisymmetric matrix, that is, the optical element is isotropic), 
the output field does not contain the opposite CP light; otherwise, the output field always contains the opposite $\mathrm{CP}$ light, and the phase delay of the opposite CP light is exactly equal to twice the orientation angle of the optical element. As a result, when CP light passes through the anisotropic material, its polarization state will change. Specifically, output light can be decomposed into two CP light beams with different handedness, and the part with the opposite handedness of incident CP light carries phase delay. In a word, for anisotropic materials, when CP light is incident, the phase delay $\varphi$ is linearly related to the orientation angle $\alpha$ of the optical element, and the relationship is as follows:

$$
\varphi= \pm 2 \alpha
$$

where the positive and negative signs indicate the handedness of incident $\mathrm{CP}$ light. In particular, when $A=1$, $B=C=0$, and $D=-1$ (corresponding to an ideal half-wave plate), the copolarization and cross-polarization conversion efficiencies are $|p|^{2}=0$ and $|q|^{2}=1$, respectively. That is to say, if CP light is incident on a nanostructure acting as an ideal half-wave plate, output light is all opposite CP light with $\pm 2 \alpha$ phase delays. It is worth noting that the half-wave plate is different from other anisotropic elements. When CP light passes through other anisotropic elements with an orientation angle of $\alpha$ with the $x$-axis, there are both parts with the same and opposite handedness of output light. However, only the part with opposite handedness has a phase delay of $\pm 2 \alpha$, so only the part with opposite handedness is the part of interest. Therefore, compared to other anisotropic nanostructures, using halfwave plate to manipulate the phase of incident light has at least two advantages. First, it does not need to remove the output $\mathrm{CP}$ light with the same handedness as incident light without phase delay, which can simplify the optical path. Second, it can improve the cross-polarization conversion efficiency. Theoretically, it can achieve lossless cross-polarization conversion and improve the energy of the polarized light participating in phase manipulation. In addition, as $\mathrm{d}|q|^{2} / \mathrm{d} \delta=\sin \delta / 2$, if $\delta=\pi+\Delta \delta$ in the case of a half-wave plate, that is, there is a small phase deviation $\Delta \delta$ between the nanostructure and a half-wave plate, the cross-polarization conversion efficiency will not decrease sharply.

Based on the principle of geometric phase, the geometry of the nanostructure is optimally designed to realize the functionality of an efficient half-wave plate. Then, the orientation angle $\alpha$ of the nanostructure is reconfigured to realize the point-by-point and precise phase manipulation of incident light. This is the basis of designing phase-only optical elements using GEMS, which is also the main content of this section.

The phase manipulation with GEMS is related to the polarization state of incident light, i.e. the sign in Eq. (5) depends on the handedness of incident $\mathrm{CP}$ light. Although this will increase the complexity of optical path, i.e. it is necessary to use polarizers, wave plates, and other optical devices to convert the unpolarized incident light into CP light before it passes through GEMS. However, the characteristics of phase control related to the polarization state of incident light also provide a design degree of freedom for the manipulation of light wavefront. For example, when the polarization state of incident light switches between left-handed circular polarization (LCP) and righthanded circular polarization (RCP), the same metalens can play the role of beam convergence and divergence, respectively. This degree of freedom has been applied to a variety of new devices and applications, such as information multiplexing, dual FOV zoom lens, multiple focal plane imaging, information encryption, etc.

As early as 2002, Bomzon et al. designed a P-B phasebased optical element using a subwavelength grating that can be equivalent to a half-wave plate [63]. Continuous phase manipulation was realized by reconfiguring the orientations of the gratings, and the influence of polarization states of incident light on the diffraction results was analyzed. In 2004 and 2005, respectively, Levy et al. designed a beam splitter with similar structures [64] and a metahologram at a working wavelength of $1.55 \mu \mathrm{m}$ [65]. In their design, each pixel of the subwavelength gratings contains multiple grating periods, and the cell size is larger than the working wavelength. Although the grating-structured element is not a metasurface in a strict sense, it is proven that it is feasible to use micro/nanostructures to realize the geometric phase control.

\subsubsection{P-B phase with metals}

In 2012, Huang et al. designed a metallic nanorod structure to build a GEMS grating (Figure 7A). The experimental results indicate that the diffraction angle of the grating is the same as that calculated by the generalized law of refraction and reflection [66]. Using this nanostructure, vortex beam is generated, and the broadband characteristics in the range of $670-1100 \mathrm{~nm}$ are proven. Subsequently, the metallic nanorods were used to build 3D GEMS holograms (Figure 7B) [67]. Due to the continuous phase modulation at the subwavelength scale, the 3D holograms have the characteristics of high resolution, large FOV, and no high diffraction orders. In addition to the use of metallic 

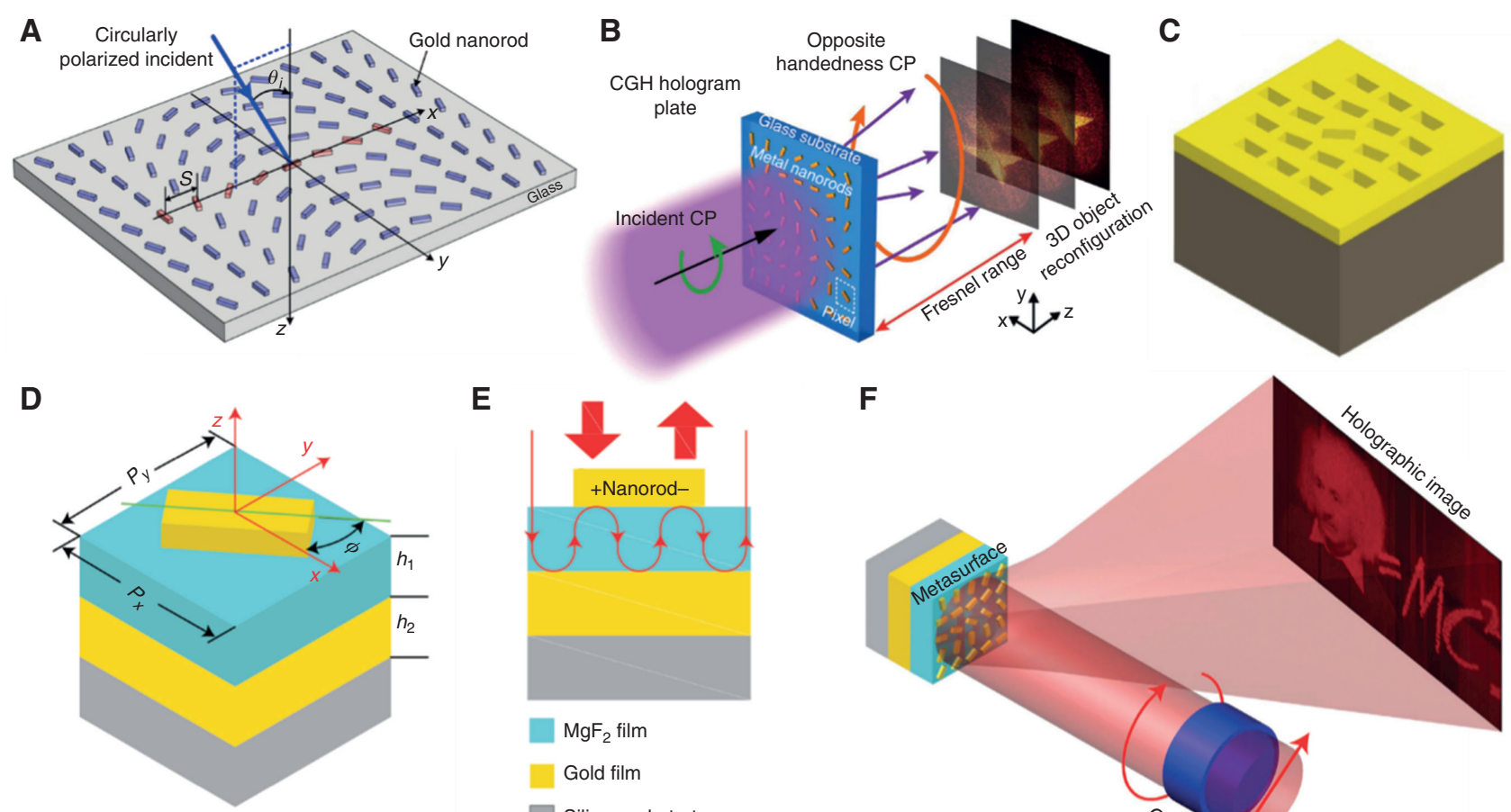

E



$\mathbf{F}$

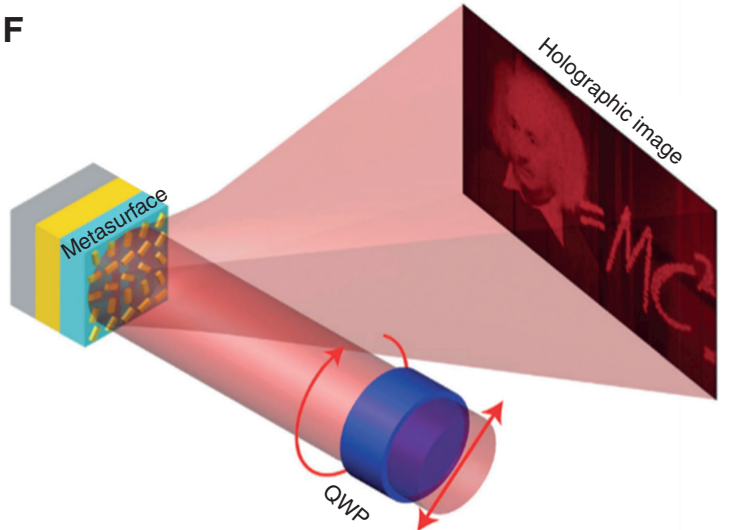

Figure 7: Illustration of geometric metasurfaces based on metallic nanostructures.

(A) Metallic nanorods with different orientations to generate geometric phases [66]. (B) Metallic nanorods to generate 3D GEMS holograms [67]. (C) Metallic hole nanostructures [68]. (D-F) MIM structure to build high-efficiency GEMS and its application in holography [69]. Reprint permission obtained from [66-69].

nanorods, the metallic hole nanostructures can also be used for geometric phase manipulation (Figure 7C). The plane Bessel lens was designed and fabricated by Gao et al. [68]. Compared to traditional lens, the image formed by the Bessel lens has a higher resolution.

The metallic nanostructures with a single-layer design mentioned above can be equivalent to polarizers. When the Jones matrix of an ideal polarizer is introduced into Eqs. (3-4) $(A=1, B=C=D=0)$, the maximum polarization conversion efficiency is only $25 \%$. That is to say, due to the limitation of working principle, the efficiency of single-layered metallic nanostructures cannot be broken through.

In 2015, Zheng et al. designed a GEMS with metalinsulator-metal (MIM) sandwiched nanostructures (Figure 7D-F) [69]. Among them, the bottom metal layer acts as a mirror, the middle dielectric isolation layer is equivalent to a F-P cavity, and the upper metallic nanorods are equivalent to nanopolarizers. As a result, incident light linearly polarized along the long axis of the nanorod is directly reflected, and that along the short axis of the nanorod enters the F-P cavity and is reflected by the bottom reflector. The two beams produce a phase difference of $\pi$, which makes the nanostructure equivalent to a half-wave plate. The measured diffraction efficiency of a complex hologram designed with MIM nanostructures can reach $80 \%$ in the near-IR band, which indicates a feasible way for the study of high-performance metasurfaces. However, the analysis shows that the device still has ohmic loss of not less than 15\%. Because the ohmic loss widely exists in metallic materials and is unavoidable, it is difficult to further improve the efficiency of metallic GEMS. Therefore, many scientists focus on the research of dielectric GEMS to pursue higher efficiency than metallic GEMS.

\subsubsection{P-B phase with dielectrics}

As a kind of material with large refractive index, silicon is promising for the design of nanostructures with large anisotropy. In 2014, Khorasaninejad et al. designed a beam splitter using silicon nanofins (Figure 8A) at a wavelength of $975 \mathrm{~nm}$ [70]. As the silicon nanofin has different equivalent refractive index along the long and short axes, a phase delay of nearly $\pi$ can be achieved. By selecting appropriate geometric dimensions, the nanofin can be equivalent to a half-wave plate. When the orientation 
A

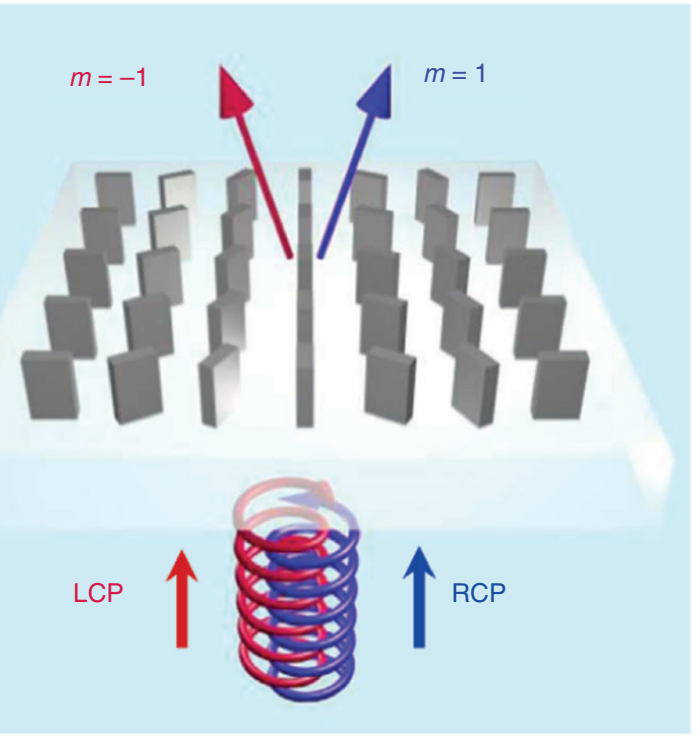

B

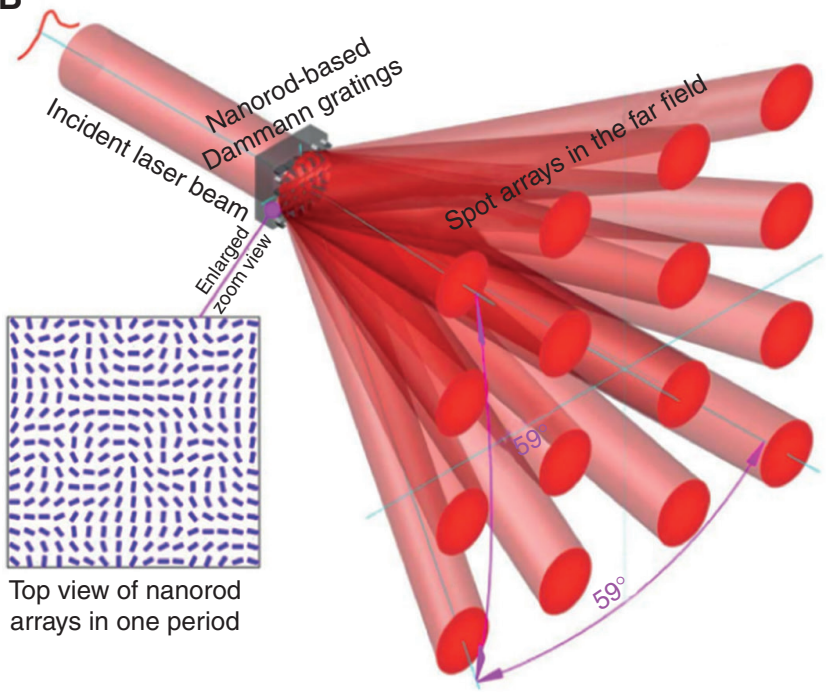

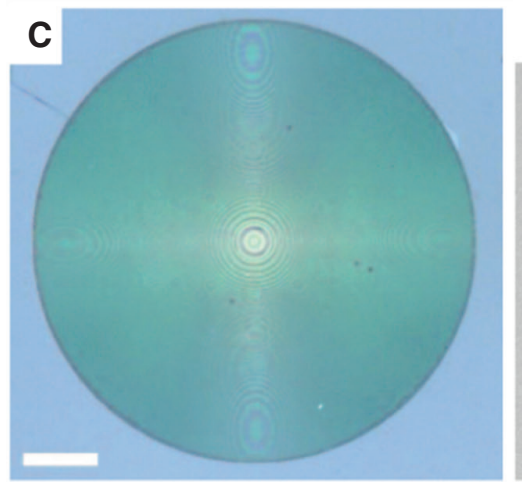

$\mathbf{F}$

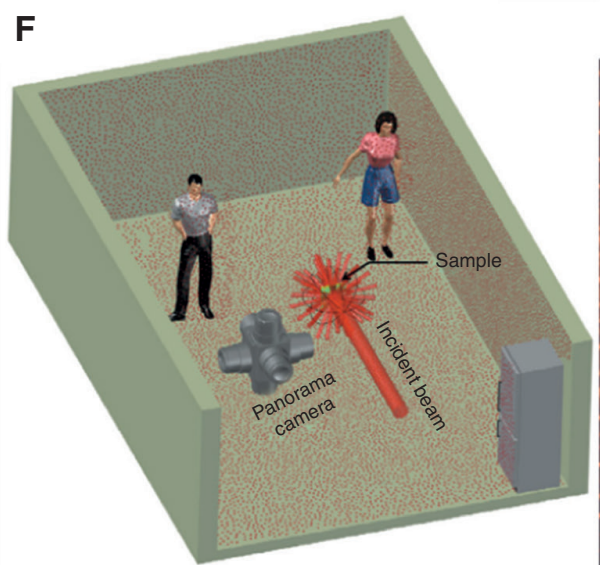

D



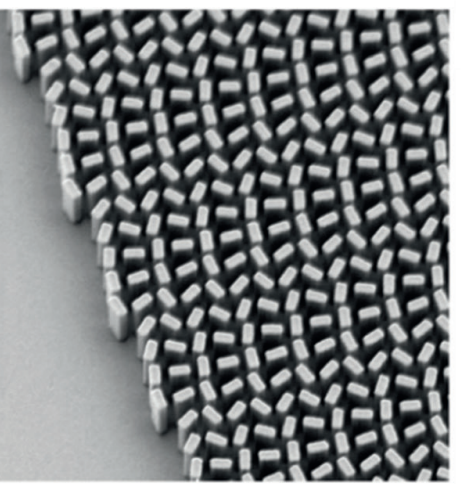

G

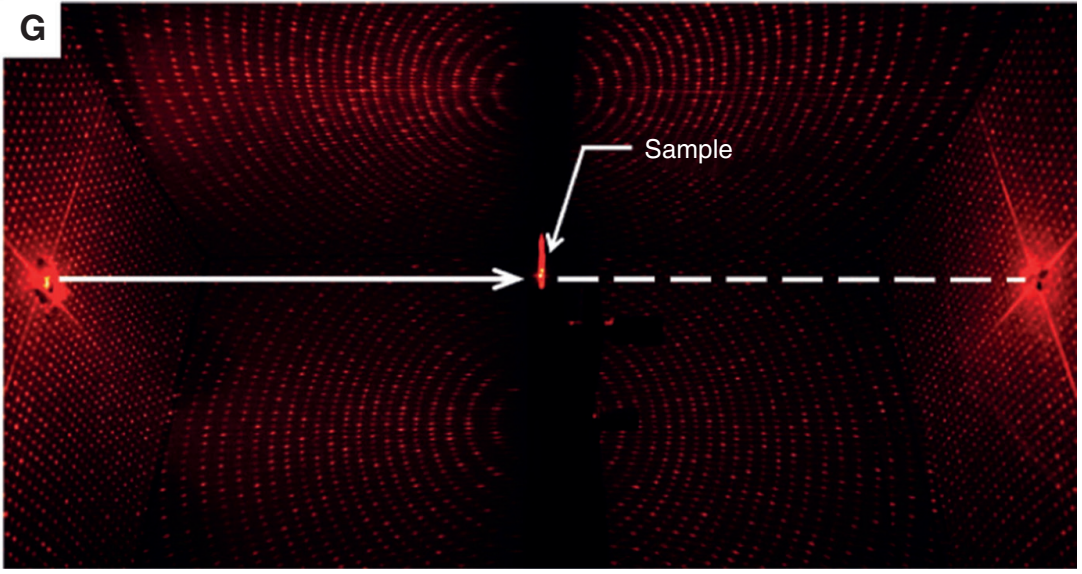

Figure 8: Illustration of geometric metasurfaces based on dielectric nanostructures.

(A) Helicity-dependent dielectric metagrating [70]. (B) $4 \times 4$ all-silicon beam splitter (Dammann grating) [71]. (C and D) Metalens with large NA based on $\mathrm{TiO}_{2}$ nanofins [72]. (E) Unit cell of a dielectric metasurface based on Mie resonance [73]. (F and G) Random point cloud generator working in $4 \pi$ space based on resonant dielectric metasurfaces [74]. Reprint permission obtained from [70-74].

angles of the arrayed nanofins increase linearly with the position coordinate, a metasurface-based blazed grating can be obtained. According to the geometric phase characteristic, the diffraction orders are opposite when $\mathrm{CP}$ light with different handedness is incident. The silicon nanofins have to sit on a transparent substrate such as silica. Therefore, to fabricate such a metasurface, one should coat a layer of silicon film on the substrate as the first step of process. In 2015, Li et al. investigated the feasibility to design all-silicon GEMS and numerically 
demonstrated its functionality by designing a $4 \times 4$ beam splitter (Figure 8B) [71].

In the visible light band, Lin et al. demonstrated the lens, grating, axicon mirror, and other functional elements using ultrathin silicon GEMS (only $100 \mathrm{~nm}$ in thickness) [75]. However, the inherent loss of silicon in visible light limits the efficiency of GEMS. In 2016, the Capasso research group from Harvard University designed a metalens with a NA of 0.8 , a diameter of $250 \mu \mathrm{m}$, optical efficiencies of $66 \%-86 \%$, and diffraction-limited resolution in visible light (Figure $8 \mathrm{C}$ and D) [72]. This is the first time to report successful high-efficiency GEMS-based metalens, which verifies the possibility of GEMS to achieve high-resolution and ultracompact optical imaging. This achievement was selected into the top 10 scientific breakthroughs of Science magazine in 2016. In this scheme, $\mathrm{TiO}_{2}$ nanostructures with high aspect ratios and high polarization conversion efficiency were fabricated by the ALD process, which raised a research upsurge of GEMS in visible light. In the same year, Devlin et al. used $\mathrm{TiO}_{2}$ nanostructures to design broadband, high-efficiency, and highfidelity GEMS holograms [76], demonstrating the ability of GEMS to realize complex wavefront control in visible light. Grover et al. set GEMS on both sides of a planar substrate [77] and realized a metalens with a NA of 0.44 and a large FOV of $50^{\circ}$ at a wavelength of $532 \mathrm{~nm}$, which has potential applications in microscopy, machine vision, etc.

Although the phase manipulation of GEMS is independent of the dimensions of nanostructures, the combination of geometric phase and Mie resonance can provide a new approach for lightwave manipulation. In 2016, Zheng et al. designed a dielectric nanostructure acting as nanopolarizer [78]. As Mie resonance occurs along the long axis of the nanostructure, it can reflect and transmit most of incident light polarized along the long and short axes, respectively. Using this nanostructure to design GEMS, the functionality of blazed grating can be realized in both reflection and refraction spaces. Furthermore, when resonances occur along both the long and short axes of the nanostructures, the nanostructures will reflect incident light polarized along both the long and short axes. At the same time, due to the existence of anisotropy, there is a certain phase difference between the two orthogonal LP light beams. Therefore, dielectric nanostructure with resonances can act as a reflective half-wave plate without metallic reflector, thus forming reflection-type GEMS (Figure 8E). In 2017, Li et al. proposed a dielectric metasurface enabled with dual magnetic resonances and studied a lightwave manipulation scheme of "Mie resonance + geometric phase" [73]. The designed dielectric GEMS have not only a simple nanostructure but also an aspect ratio as low as 1.5 , which greatly reduces the fabrication difficulty of dielectric metasurfaces.

From the above analysis, when the working wavelength falls in the resonance frequency band, the dielectric metasurface shows the unusual reflection characteristic. When the working wavelength deviates from the resonant wavelength of dielectric nanostructures, a part of incident light would transmit with the same phase delay as that of reflected light. Therefore, the diffractive beams with phase delays will fill in the whole $4 \pi$ space. By flexibly designing the peak wavelength of Mie resonance of dielectric nanostructure, the ratio of light energy between transmission and reflection can be effectively controlled. Based on this, a more generalized "arbitrary energy ratio of reflection/ refraction + geometric phase" approach for light manipulation is proposed [74]. Based on this principle, a random point cloud generator that can work in the $4 \pi$ space is experimentally demonstrated (Figure $8 \mathrm{~F}$ and $\mathrm{G}$ ).

\subsection{Intensity control based on Malus' law}

In addition to the geometric phase control, the anisotropic nanostructures with varied orientations can be employed to conduct light intensity control based on Malus' law. For example, Ellenbogen et al. designed a kind of metallic cross-shaped nanostructures, and two nanoarms with different lengths have different spectral responses. Therefore, when the polarization direction of incident light varies, the metasurface can display different colors (Figure 9A) [79]. In 2018, Yue et al. used halfwave plates with different orientation angles to build a metasurface for ultracompact image display. When LP light is incident on the metasurface, light intensity distribution will be encoded into polarization profiles. With a bulky polarizer, a hidden high-resolution grayscale image can be decoded (Figure 9B) [80]. In 2019, Dai et al. proposed a high-density image display technology based on nanopolarizers (Figure 9C) [81]. The nanopolarizers are composed of silicon-on-insulator (SOI) nanobricks enabled with magnetic resonances. Very recently, Deng et al. proposed a multiplexed metasurface nanoprintings for anticounterfeiting application enabled with the orientation degeneracy of nanostructures [82]. These metasurfaces are simple and ultracompact in structures, which can overcome the inherent defects of traditional image display technology, such as complex optical system, large pixel size, and so on. Therefore, they have promising applications in the fields of high-density optical data storage, high-end anticounterfeiting, information encryption, and so on. 


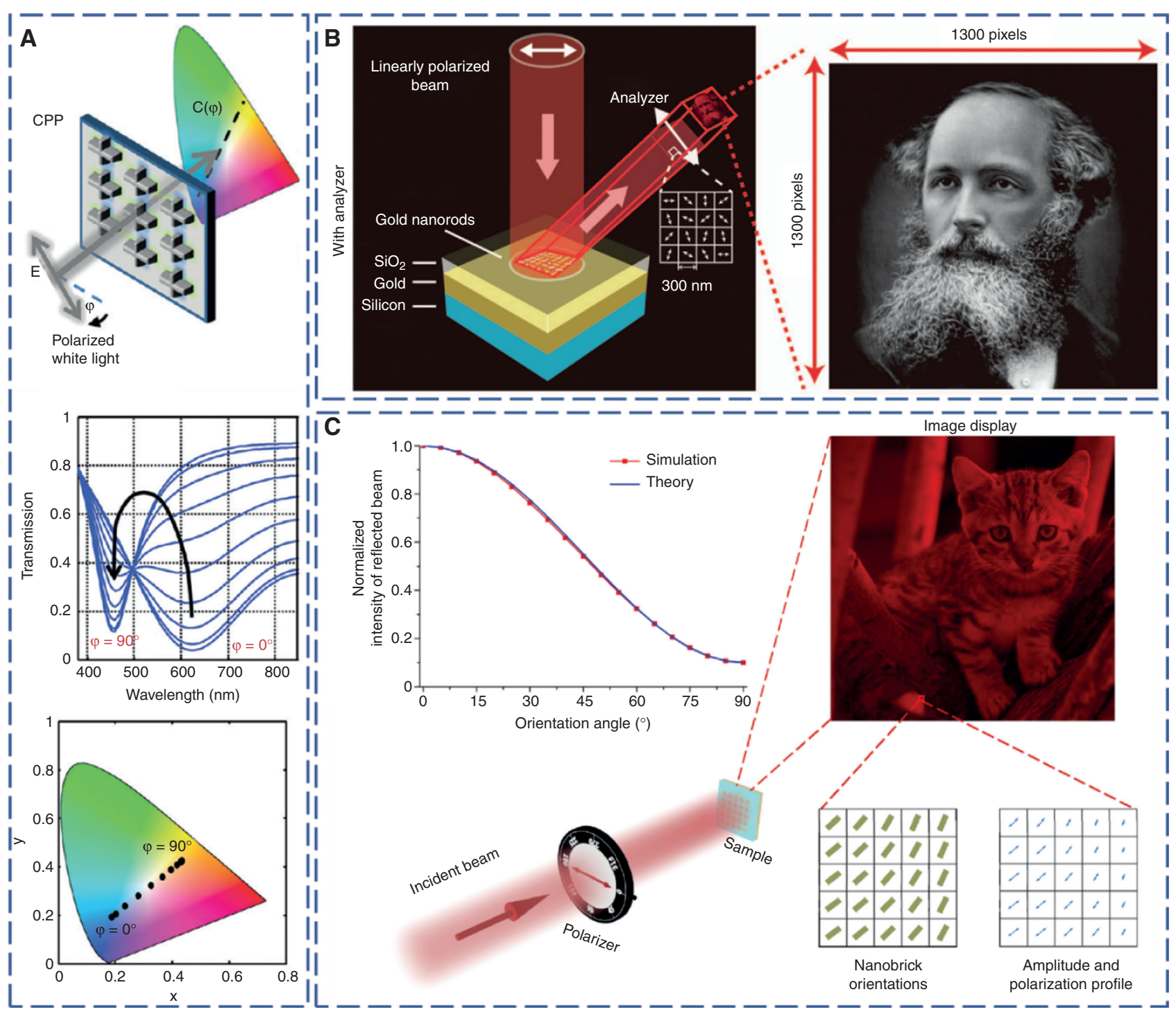

Figure 9: Metaimage displays by intensity control based on Malus' law.

(A) Cross-shaped metallic nanostructures for active color control [79]. (B) MIM nanostructures acting as half-wave plates for intensity manipulation [80]. (C) SOI nanostructures acting as nanopolarizers for intensity manipulation [81]. Reprint permission obtained from [79-81].

\subsection{Multifunction and information multiplexing}

\subsubsection{Multifunctional GEMS with helicity control}

According to the fact that the phase manipulation of incident CP light with different handedness by GEMS is opposite to each other, Chen et al. designed a dualpolarity metalens based on polarization control [83]. When incident light on the metalens changes from RCP to LCP, output light changes from the focusing state to the divergent state, and the image can be magnified and demagnified using the same metalens (Figure 10A). Using the combination of two GEMS metalenses, Zheng et al. realized a dual-FOV step-zoom metalens, while the working distance remains unchanged (Figure 10B) [84]. This kind of step-zoom metalens can readily switch between two FOVs and has potential application in target tracking and recognition. In 2019, Cui et al. combined the Moire effect with GEMS, and by rotating one of the two GEMS, the focal length changes from $-\infty$ to $+\infty$ theoretically (Figure 10C) [85].

\subsubsection{Multifunctional and multiplexed GEMS based on segmented and interleaved nanostructures}

Because different nanostructured unit cells of a GEMS can be independently designed for phase control of 


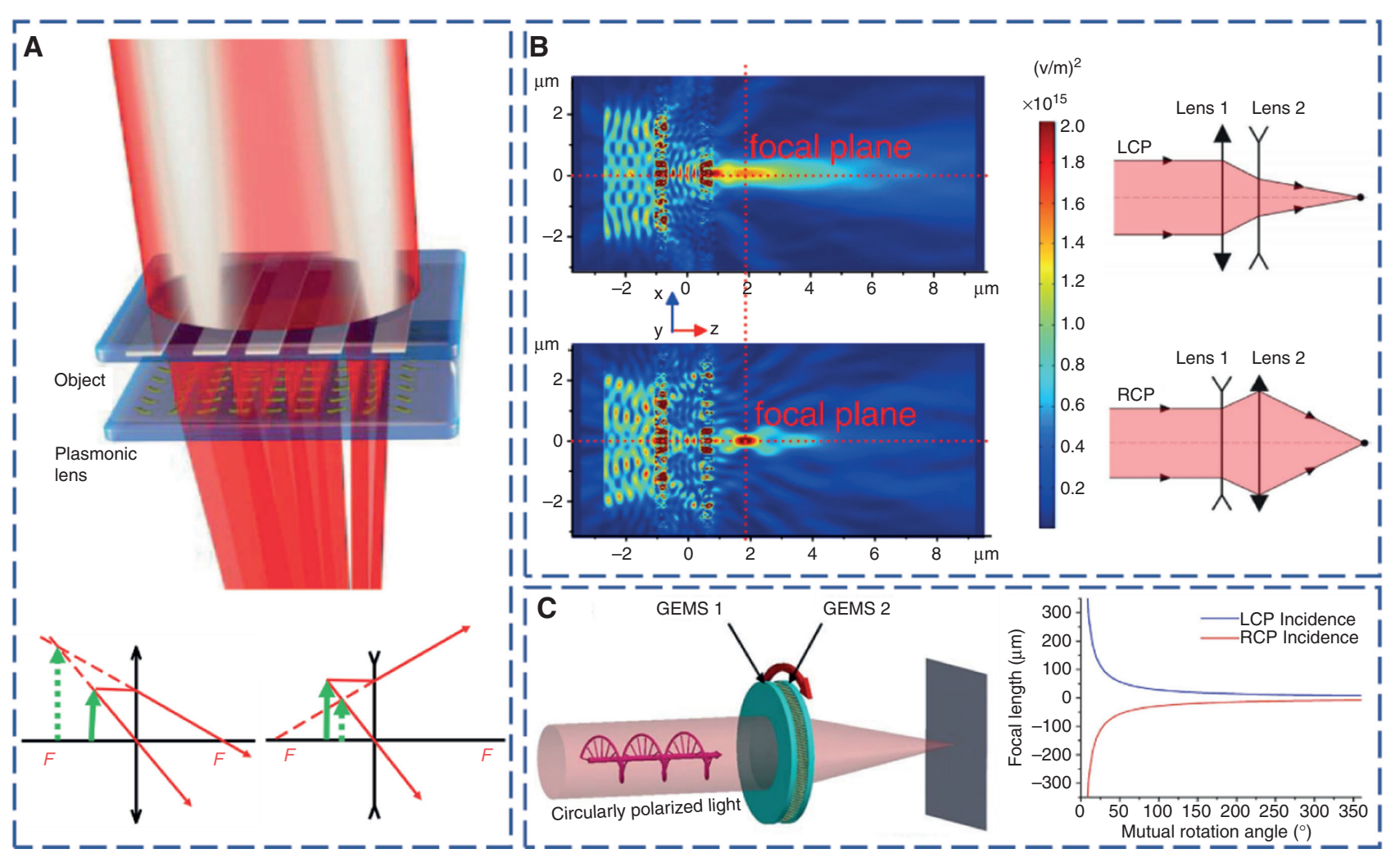

Figure 10: Multifunctional GEMS with helicity control.

(A) Metasurfaces acting as both positive and negative lenses [83]. (B) Dual FOV step-zoom metalens [84]. (C) Continuous-zoom metalens conducted with Moire effect [85]. Reprint permission obtained from [83-85].

light, it is easy to use nanostructured unit cells in different areas to achieve different functionalities. For example, Chen et al. divided a piece of metalens into three regions and designed the phase of each region according to different focal lengths, which can attain the multifocusing of light (Figure 11A) [86]. This kind of functionality is difficult to realize for conventional lens because it is very difficult to obtain different curvature radii on a lens surface with different focal lengths. Using similar approach, Mehmood et al. realized the vortex beam with different topological charges in different focal planes (Figure 11B) [87]. Wen et al. arranged the cylindrical GEMS metalens and spherical GEMS metalens alternately (Figure 11C) [88]. When changing the handedness of incident CP light, optical 1D and two-dimensional (2D) Fourier transformation can be switched. Khorasaninejad et al. designed different regions of a metasurface as different off-axis lenses (Figure 11D) [89]. One object can be imaged as two different images on the image plane through the proposed metalens. The brightness and darkness of the image indicate the chirality of the object wave. Therefore, the metalens can be used for the detection of chiral molecules.
In terms of holography, Wen et al. encoded two holograms into one GEMS with interleaved nanostructures [90]. When the handedness of incident light changes from LCP to RCP, the holographic patterns will be interchanged (Figure 11E), and the chiral multiplexing GEMS hologram has high fidelity in a wide band. In 2019, Huang et al. realized a geometric phase nanostructure with efficiency up to $80 \%$ in the ultraviolet (UV) band ( $355 \mathrm{~nm}$ ) using niobium pentoxide $\left(\mathrm{Nb}_{2} \mathrm{O}_{5}\right)$ materials [91]. A polarization multiplexing and dual-channel anticounterfeiting GEMS with two sparse metaholograms is designed (Figure 11F). This kind of GEMS, which can work in UV band, can be used in nanophotography, bioimaging, and other fields. Zhang et al. took two mutually centrosymmetric patterns as target patterns [92], designed holograms for incident CP light with different handedness, respectively, and integrated them into a GEMS (Figure 11G). This ingenious design can extract Stokes parameters of the incident polarized light. Zhang et al. combined a piece of metahologram based on geometric phase with a Malus metasurface encoded with a hidden grayscale image and realized multichannel anticounterfeiting based on polarization control [94]. Different from the geometric phase based nanostructures in the 


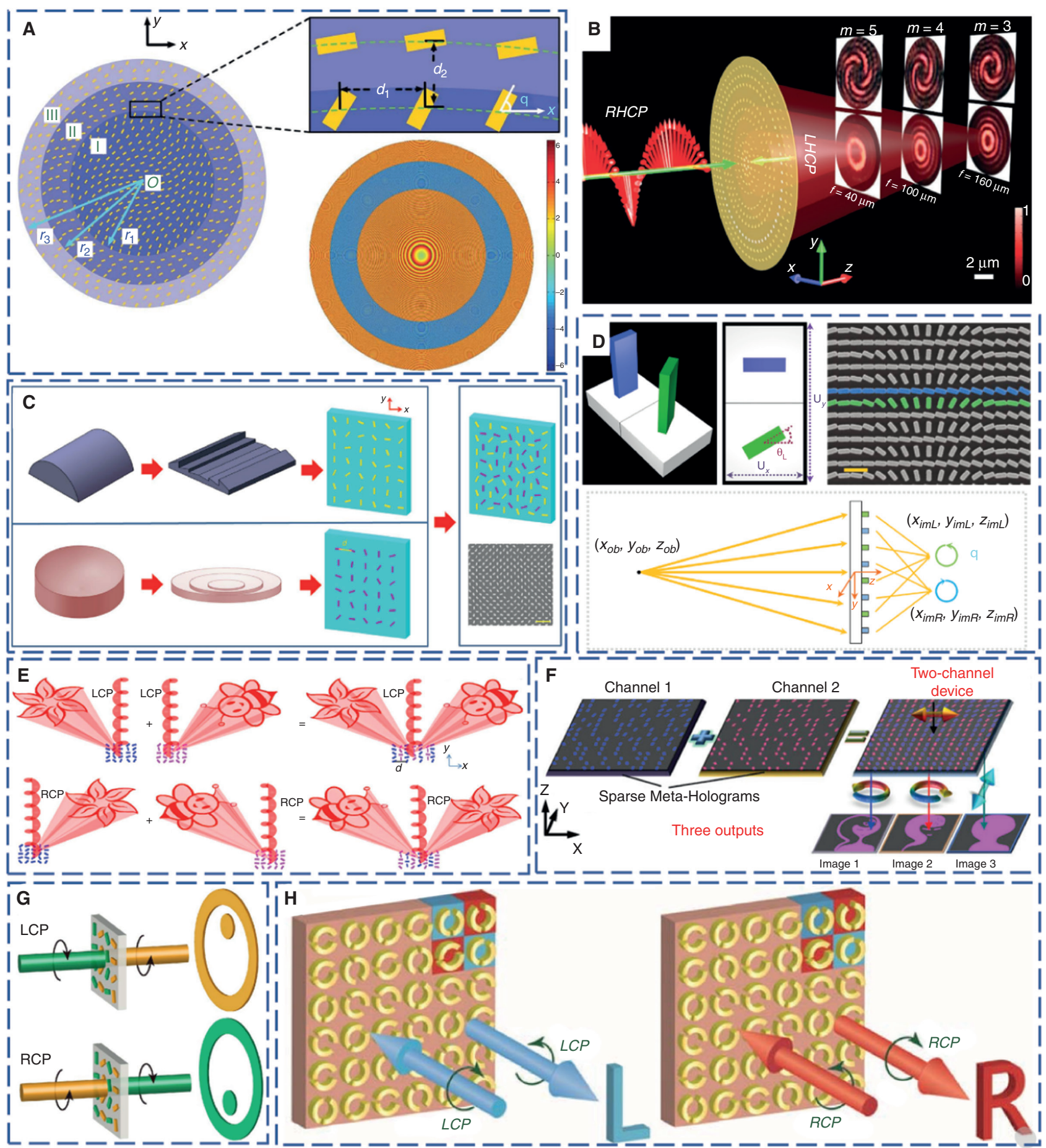

Figure 11: Segmented and interleaved nanostructures for multifunctional and multiplexed metasurfaces.

(A) Metalens to produce multifocal spots [86]. (B) Spiral phase plates for generating vortex beams with different topological charges [87]. (C) Integration of cylindrical and spherical metalenses for 1D and 2D Fourier transformation [88]. (D) Integration of dual-off-axis metalenses [89]. (E-G) Dual-mode metaholograms [90-92]. (H) Two kinds of nanostructures that can manipulate the phase of RCP or LCP light independently [93]. Reprint permission obtained from [86-93].

above work, Wang et al. designed two different nanostructures to manipulate the phase of RCP and LCP light independently (Figure 11H) [93] rather than the opposite phase control of two CP light with different handedness. This kind of CP-selective metasurface can reduce the interference of light with different handedness to the diffraction results. 


\subsubsection{Wavelength-selective metasurfaces for color holography and printings}

If one can optimize the geometry of nanostructures with narrow-band response of red, green, and blue light, respectively, and integrates these nanostructures with different dimensions into a supercell, it is promising to provide an approach for compact color image displays. In 2015, Zhao et al. designed a supercell with dozens of unit cells to reduce the near-field coupling effect after the optimization of three-color narrowband nanostructures (Figure 12A and B) [95] and verified the color holography by numerical simulations. Because the size of the supercell is close to $10 \mu \mathrm{m}$, the diffraction angle of the hologram is only a few degrees. To realize color metaholography, Wang et al. designed an ultracompact supercell (Figure 12C) with a unit cell size of only $420 \mathrm{~nm}$, and the experiment verified the design [96]. Then, they used a reconfigurable metasurface to build a color hologram by changing the polarization state of incident light (Figure 12D) [97]. Different from holography, Zang et al. used a metasurface to encode the intensity of color components into polarization profiles. When incident light passes through the metasurface, it can be decoded by an analyzer to obtain the color image with high resolution, high fidelity, and no color crosstalk (Figure 12E) [98]. In 2019, Wei et al. used two kinds of nanostructures with different spectral responses to build a two-color printing pattern with white light incidence. At the same time, when a broadband laser source with two different wavelengths of 540 and $645 \mathrm{~nm}$ illuminates the same metasurface, a colorful holographic pattern can be reconstructed in the far field (Figure 12F) [99].


$\mathbf{F}$
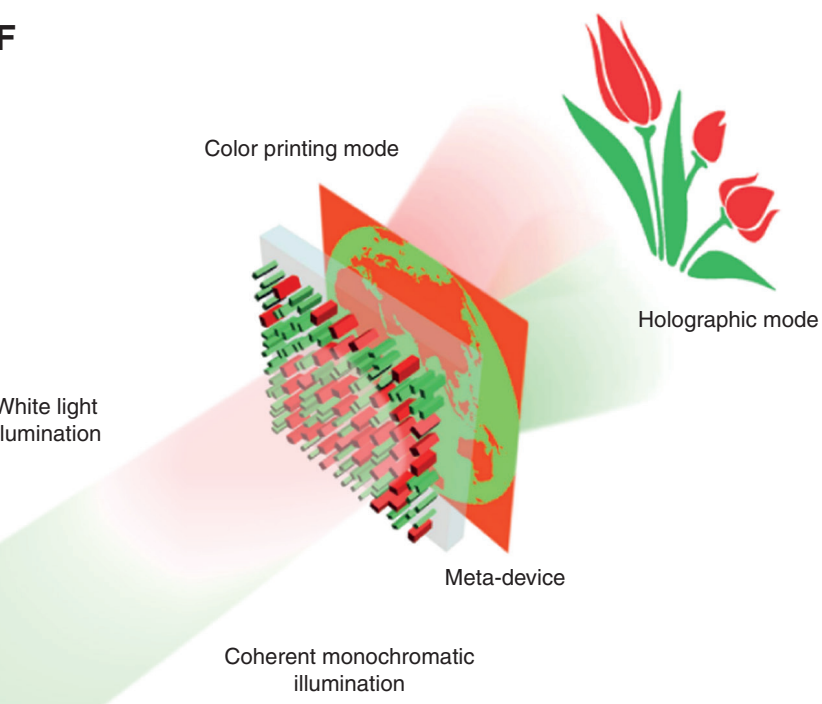

Figure 12: Wavelength-selective metasurfaces for color holography and printings.

(A and B) Supercell design with three kinds of nanostructures for color holograms [95]. (C) Ultracompact supercell to produce different spectral responses for red, green, and blue light [96]. (D) Color-varied hologram with different helicity of incident light [97]. (E) Two-color printings with silicon nanobricks [98]. (F) Combination of printings and holography with a single metasurface [99]. Reprint permission obtained from [95-99]. 


\subsubsection{Information multiplexing based on the "many-to-many" scheme}

The above-mentioned approaches with segmented or interleaved nanostructures can be called a "one-to-one mapping" scheme: each nanostructure responses to one specific wavelength and contributes to one holographic/ nanoprinting pattern. Except for it, there is also a "manyto-many" scheme that can be used for information multiplexing, that is, each holographic pattern is obtained by the collaboration of all nanostructures. The scheme can be realized in three different ways: (1) at the incident light end, changing the phase distribution of the incident wavefront on the metasurface; (2) at the transmission end, changing the diffraction characteristics of light with different wavelengths or handedness on the metasurface; and (3) at the observation end, changing the observation conditions in the observation plane.

In terms of the incident end, Zhang et al. studied the influence of incident angle on the diffraction results of GEMS, designed different holograms according to different incident angles (Figure 13A), and obtained eight-channel 2D image multiplexing and two-channel 3D image multiplexing [100]. Li et al. and Wan et al. proposed a metasurface composed of metallic nanoholes [101, 104]. With red, green, and blue light incident at different angles, 3D
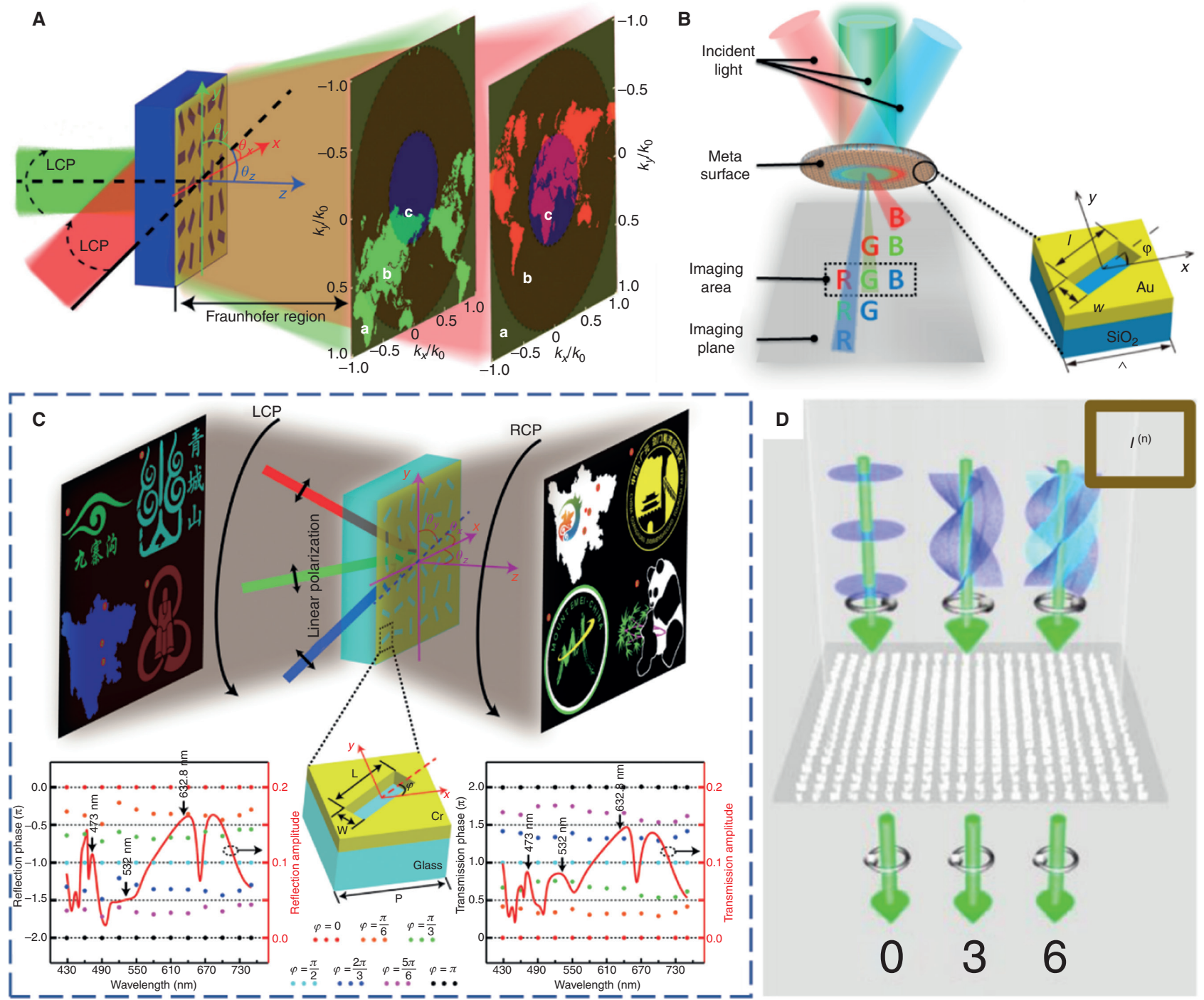

Figure 13: "Many-to-many" scheme for information multiplexing in terms of the incident end.

(A) Multiplexed holograms with varied incident angles [100]. (B) Color holography using different incident angles for red, green, and blue light [101]. (C) Color holography in both transmission and reflection spaces using different incident angles for red, green, and blue light [102]. (D) Multiplexed metahologram using different topological charges [103]. Reprint permission obtained from [100-103]. 
color holography is realized (Figure 13B). Furthermore, at oblique incidence, the phase delays of LCP light reflected by GEMS and RCP light transmitted by GEMS are no longer opposite to each other, and hologram in the reflection and refraction spaces can be designed independently. Based on this principle, Zhang et al. optimized the orientation angles of metallic nanoholes according to the target holographic patterns (Figure 13C). With oblique incidence of red, green, and blue light, they obtained two independent color holograms in both reflection and refraction spaces [102]. As both incident angle and topological charges will change the phase distribution of the wavefront on a metasurface, multiplexed vortex beams can be obtained. In 2019, Jin et al. designed a GEMS for orbital angular momentum (OAM) multiplexing [103]. When the OAM of incident light is different, the reconstructed holograms are also different (Figure 13D).

In the transmission end, the phase delay is related to the handedness of incident CP light, whereas Fresnel diffraction is related to the wavelength. Using these features, Jin et al. proposed a multiplexed metasurface with threewavelength, dual-chiral, and noninterleaved design. Theoretically, there are six independent incident modes (Figure 14A). By combining these modes, $2^{6}-1$ holographic patterns can be generated [105]. Ye et al. used GEMS to control the nonlinear effect of lightwave for holographic image multiplexing. When CP light is incident on metallic nanostructures with an orientation angle of $\alpha$, output light is composed of three parts: light with the same frequency, opposite handedness, and phase delay of $2 \alpha$; light



D

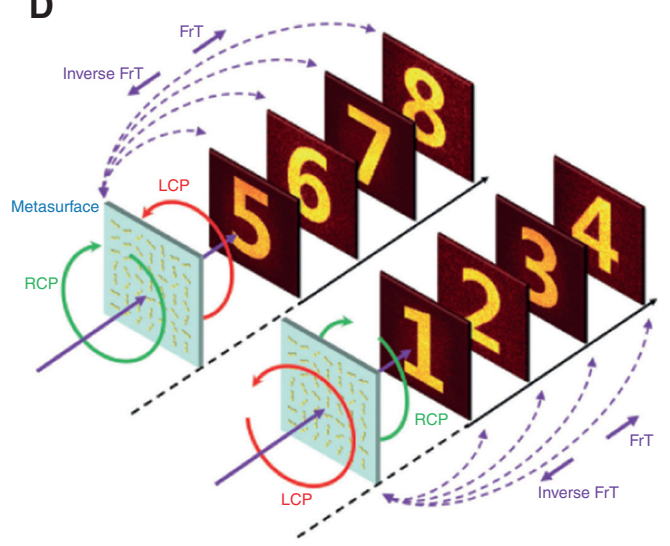

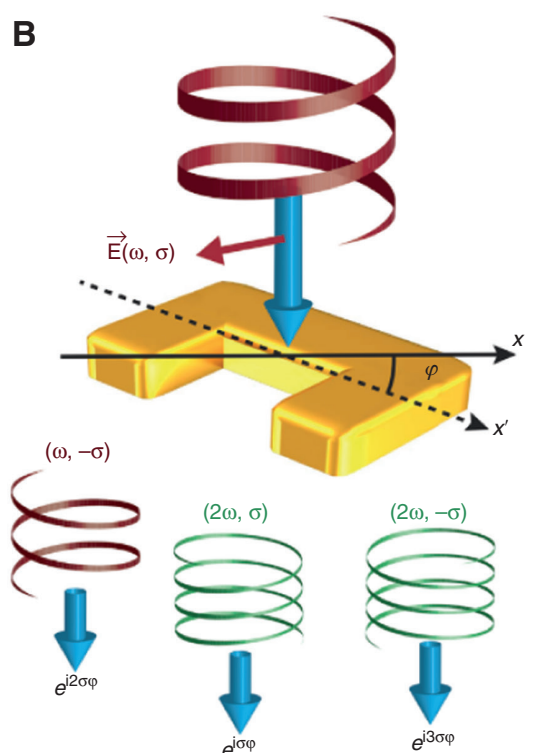

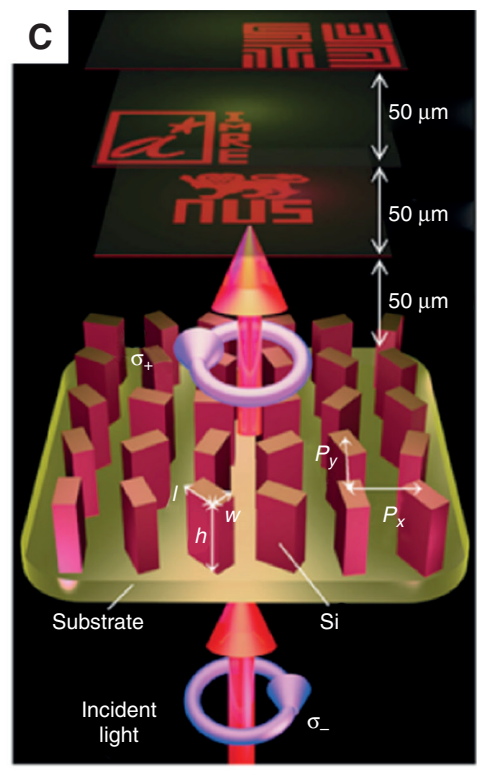

E
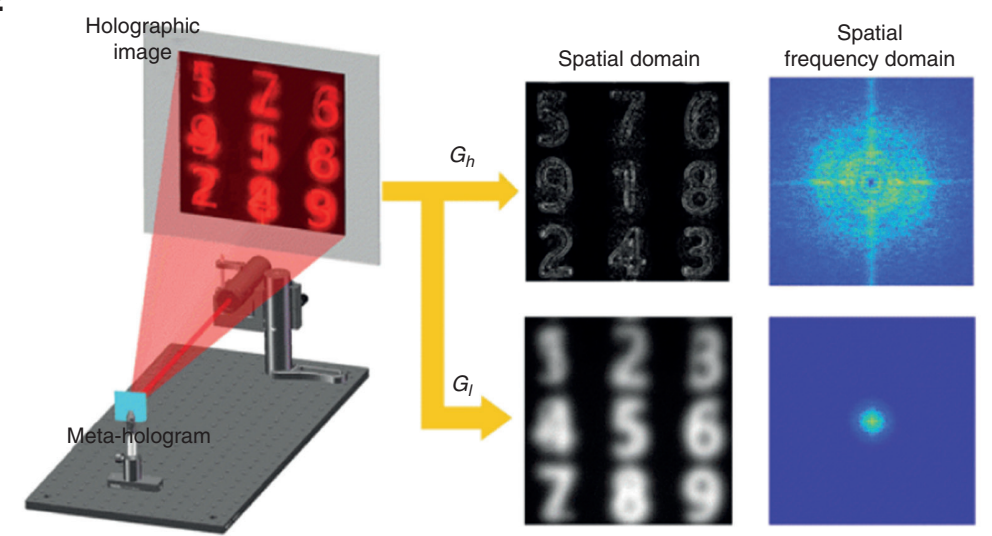

Figure 14: "Many-to-many" scheme for information multiplexing in terms of the transmission and observation ends.

(A) Multiplexed metasurfaces by taking wavelength and helicity as design degrees of freedom [105]. (B) Multiplexed metasurfaces enabled with nonlinear effect [106]. (C and D) Multiplexed Fresnel holograms by assigning different working distances [107, 108]. (E) Metasurfaces with spatial frequency multiplexing [109]. Reprint permission obtained from [105-109]. 
with twice the frequency, same handedness, and phase delay of $\alpha$; and light with twice the frequency, opposite handedness, and phase delay of $3 \alpha$ (Figure 14B). Using this nanostructure to design a GEMS, holographic image multiplexing with three channels is realized [106].

In the observation end, as the Fresnel holographic pattern is usually designed at a fixed working distance, using optimization algorithm to design the orientations of the nanostructures, different target patterns can be obtained at different observation distances. Huang et al. and Wei et al. realized the above functionalities with dielectric and metal-based metasurfaces, respectively (Figure 14C and D) [107, 108]. In 2019, Deng et al. proposed a metasurface based on spatial frequency multiplexing [109]. Two completely independent images can be overlapped at the same time using different spatial frequency information and recorded on a metasurface. When different spatial filters are set on the observation, the two images can be separated (Figure 14E).

In recent years, a unit cell composed of two identical nanostructures has attracted the attention of researchers [110-116]. Lee et al. used a pair of nanorods to build an X-shaped metasurface. By adjusting the two orientation angles of the two nanorods, they can modulate the complex amplitude of incident lightwave (Figure 15A) [110]. The ability of complex amplitude control is conducive to complex holographic design such as 3D holograms, and different holographic patterns are experimentally obtained on different diffraction planes. Deng et al. fixed the relative orientation angle of a pair of nanorods to $\pi / 2$. By changing the center position of nanorods, not only the holographic pattern with specific intensity distribution

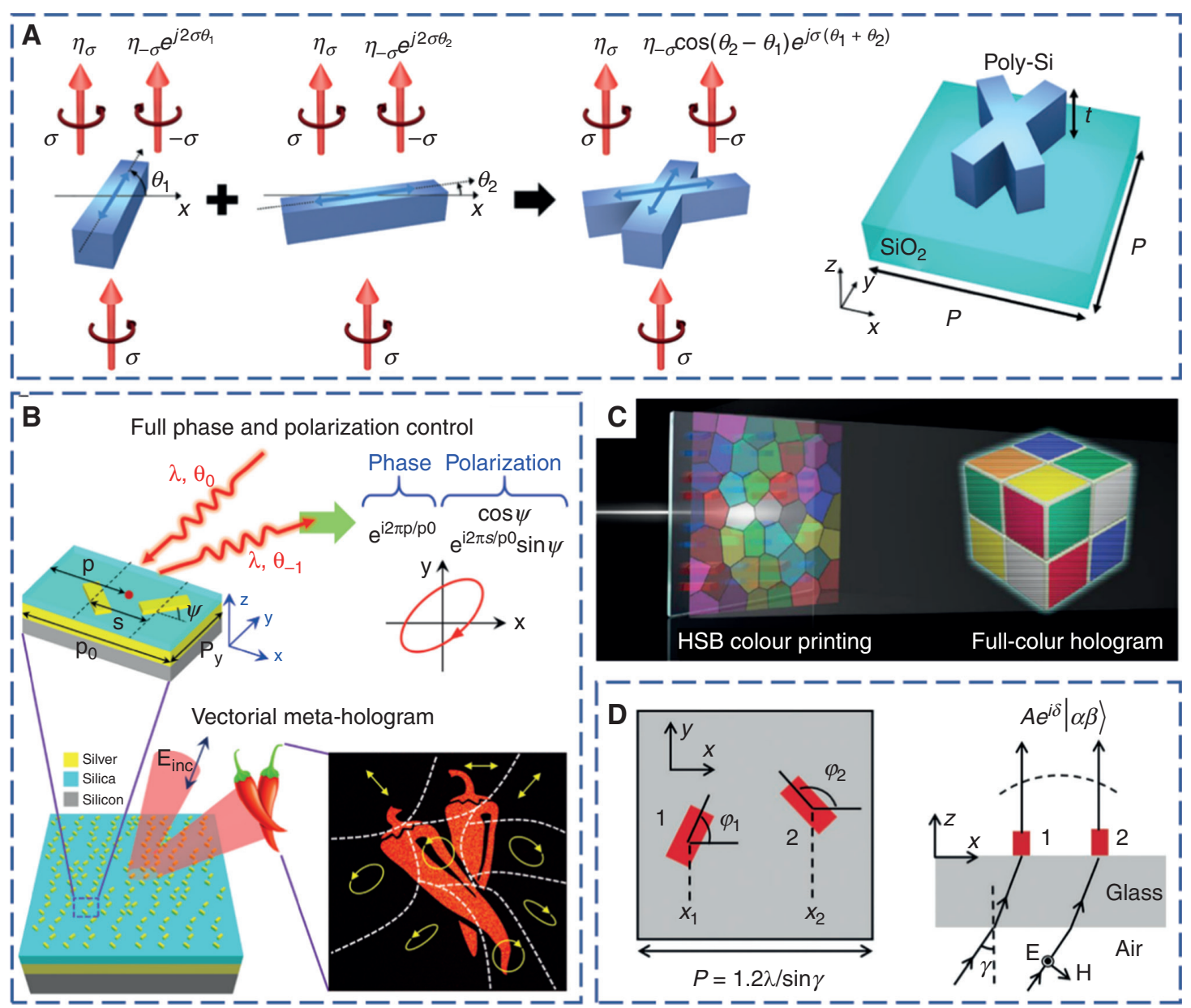

Figure 15: Metasurfaces with a unit cell composed of two identical nanostructures.

(A) Complex amplitude manipulation based on X-shaped nanostructures [110]. (B) Unit cell composed of two identical but separate nanorods for manipulation of the phase and polarization state of light simultaneously [111]. (C) Complex amplitude manipulation with a pair of nanostructures to conduct simultaneous full-color holography and HSB color printings [115]. (D) Unit cell composed of two identical but separate nanostructures for manipulation of intensity, polarization states, and topological charges of the vortex beam simultaneously [116]. Reprint permission obtained from $[110,111,115,116]$. 
can be realized but also the polarization state of the light field can be controlled (Figure 15B) [111]. The polarization encryption property of the vectorial holographic metasurface can increase the security of an encoded image. Bao et al. combined the complex amplitude manipulation ability of a pair of nanostructures with the narrowband response characteristics of red, green, and blue light and produced color printing images in the near field and color holograms in the far field simultaneously (Figure 15C) [115]. Arbitrary hue saturation brightness (HSB) control can be realized in the two color images. By changing the orientation angles and center positions of the two nanoblocks at the same time (Figure 15D), they attained vortex beams with arbitrary intensity distribution, polarization characteristics, and topological charges [116]. The strong control of lightwave can also be used to realize information multiplexing.

\subsection{Applications}

In addition to the fundamental optical elements such as lenses, gratings, spiral phase plates, printings, and holograms, there are also some unique applications of metasurfaces composed of nanostructures with varied orientation angles. Because GEMS can precisely control the phase of lightwave point by point, it can be employed to realize some special light fields and measure the spectrum, polarization state, and displacement. For example, off-axis lens with a large off-axis amount can be attained without increasing the manufacturing complexity using GEMS. Based on this, Khorasaninejad et al. designed an off-axis metalens with an off-axis angle of $80^{\circ}$ [117]. More importantly, GEMS can manipulate the phase exactly twice of the nanostructure orientation angle, which is independent of the wavelength and has a broadband characteristic. Therefore, the off-axis metalens can focus incident light in the broad range. According to the relationship between focal length and wavelength, the focal positions are different when light with different wavelengths is incident (Figure 16A). Therefore, the off-axis lens can be used for spectral measurement, and the wavelength resolution in the optical fiber communication band is up to $200 \mathrm{pm}$. Liu et al. designed and experimentally demonstrated a planar Cassegrain-Schwarzschild objective with GEMS [126]. Chen et al. proposed a spectral tomographic imaging technology based on aplanatic metalens by focusing different wavelengths on different focal planes [127]. Yang et al. measured the phase gradient and polarization distribution of incident lightwave using GEMS-based metalens arrays (Figure 16B) [118]. This compact Hartmann-Shack wavefront sensor can be used to measure vectorial beam and vortex beam in real time. In 2019, Yuan et al. studied the generation of superoscillatory optical fields using GEMS, which can obtain a very high resolution $(-\lambda / 100)$ near the superoscillatory hotspot (Figure 16C) [119]. In the same year, they published their research results of GEMS applied to optical metrology [120]. They used GEMS to produce light field with extremely high phase gradients (Figure 16D), realized the displacement measurement with resolution of less than $1 \mathrm{~nm}$, and claimed that it can theoretically achieve the displacement resolution of $\lambda / 4000$ (atomic level). This is a major breakthrough in the direction of high-precision measurement based on metasurfaces, which will have a significant impact on the micromachinery, chip lithography positioning, deformation and displacement measurement, sensing, etc.

Some applications can also be realized using GEMS to adjust the optical phase of CP light with different handedness. Zhou et al. designed a GEMS grating for image edge extraction. When the object light wave passes through the metagrating, a pair of images with a small relative displacement appear. The amplitudes of the two images are opposite, so that the light intensity of the overlapping area is 0 , and the image edge extraction is realized (Figure 16E and F) [121]. Liu et al. combined the single pixel ghost imaging (GI) with GEMS (Figure 16G). When the handedness of incident CP light is different, the target GI image will change, thus affecting the reconstruction result [122]. Even if the randomly binary mask information and the detected intensity values in the single pixel are leaked, the correct reconstruction results cannot be obtained if the polarization state of incident light is unknown, which further increases the security of optical encryption technology based on GI. Yue et al. designed two asymmetric off-axis images; when LP light is incident, it can produce the optical illusion pattern of "Rubin's vase", whereas when the individual LCP or RCP light is incident, the optical illusion pattern disappears (Figure 16H) [123]. Lee et al. implemented augmented reality (AR) with GEMS lens [124]. The metalens is made of nanoimprinting technology and has a diameter as large as $2 \mathrm{~cm}$ and a wide FOV. With RCP, the metalens can focus LCP light, but it cannot manipulate the phase of RCP light. The RCP part of the reflected light from the real object is extracted and directly enters the human eyes, whereas the LCP light is composed of virtual pattern and converges into the human eyes after passing through the metalens. Therefore, the human eyes can observe the real object and the virtual object at the same time (Figure 16I). When the diffraction field of the target is centrosymmetric, GEMS is no longer sensitive to the polarization state of 

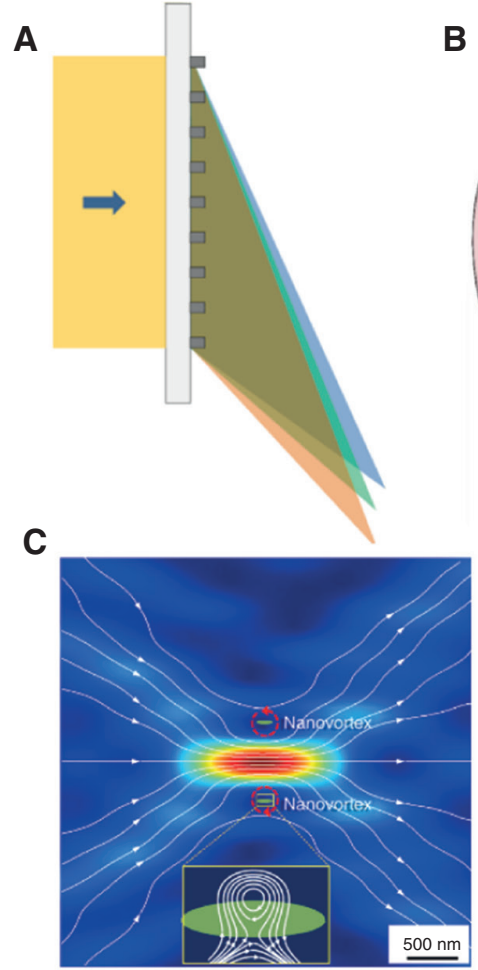

$\mathbf{E}$

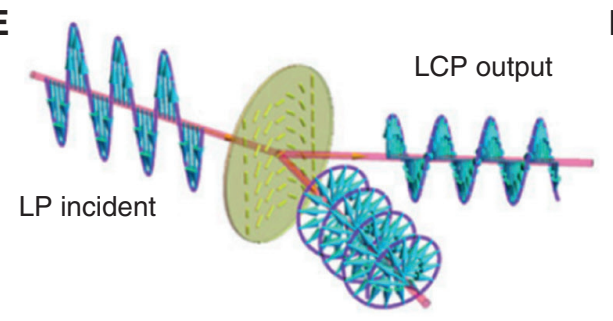

RCP output

D
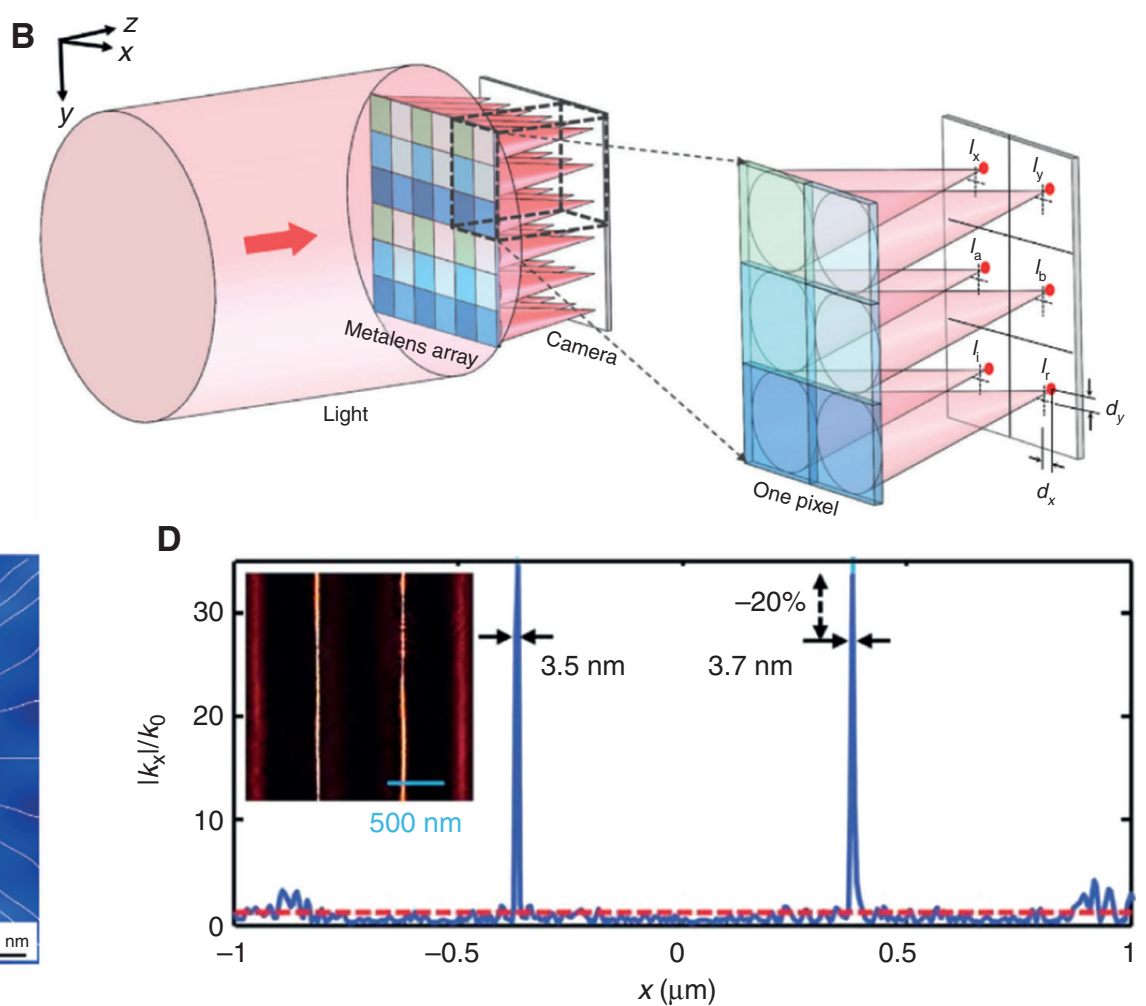

G

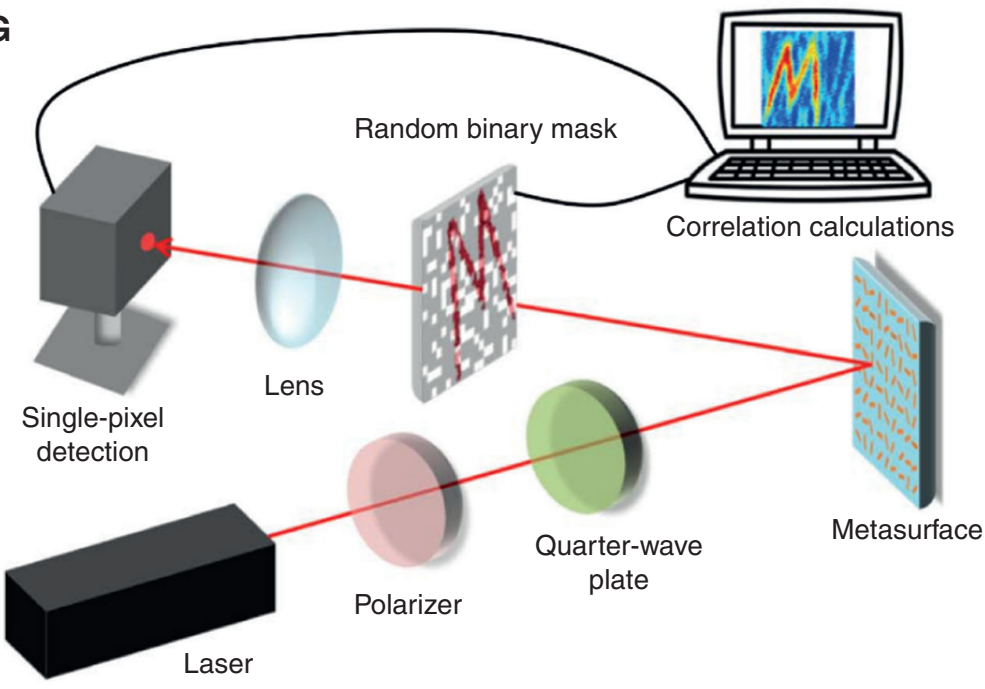

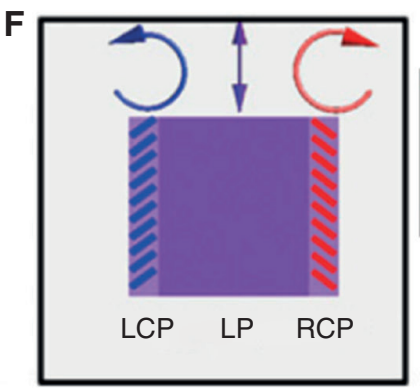

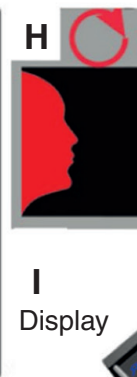





Figure 16: Application examples of GEMS.

(A) Spectral measurement [117]. (B) Polarization state measurement [118]. (C) Generation of superoscillation [119]. (D) High-precision displacement measurement [120]. (E and F) Image edge inspection [121]. (G) Single-pixel GI [122]. (H) Optical illusion [123]. (I) AR [124]. (J) Polarization-independent beam scattering [125]. Reprint permission obtained from [117-125]. 
incident light. Based on this, Xu et al. designed a broadband polarization-independent metasurface scattering device (Figure 16J) [125]. In a word, using or avoiding the polarization dependence of GEMS, a new type of metasurface-based optical elements with unique functionalities can be designed. In addition, the amplitude and polarization modulation characteristics of the metasurfaces can also be used in the field of micro/nanofabrication. For example, Yu et al. designed a nanostructure acting as a polarizer in the UV band, and the metamask composed of these arrayed nanostructures can significantly reduce the UV exposure time in the nanofabrication [128].

\section{Anisotropic nanostructures with varied dimensions and orientations as design degrees of freedom}

\subsection{Amplitude and phase control}

In the past few years, scientists have been trying to redistribute the information between different channels to increase the number of information channels through a single metasurface. Although multichannel is helpful to enrich the diversity of image display, limited by the design degree of freedom of nanostructures, the total information capacity recorded on a metasurface is difficult to improve, and the crosstalk between the channels is difficult to reduce. Therefore, the researchers considered changing both the anisotropy and the orientation of the nanostructures and proposed metasurfaces with three design degrees of freedom that can control the lightwave more flexibly. By changing the length, width, and orientation angle of nanofins, complex amplitude control can be conducted (Figure 17A). Based on the principle, Song et al. realized complex amplitude modulated holography [129]. Overvig et al. employed a metasurface to generate a grayscale pattern on the sample surface and a hologram in the object plane simultaneously or two different holograms when illuminating the metasurface with two different wavelengths (Figure 17B) [130]. Yoon et al. designed two kinds of nanostructured unit cells [131]. When illuminated with a wide spectral light source, the two unit cells show different colors due to different spectral responses. When the $635 \mathrm{~nm}$ CP laser beam is incident, the two unit cells have the same geometric phase control characteristics, which can generate holographic pattern in the far field (Figure 17C).

A

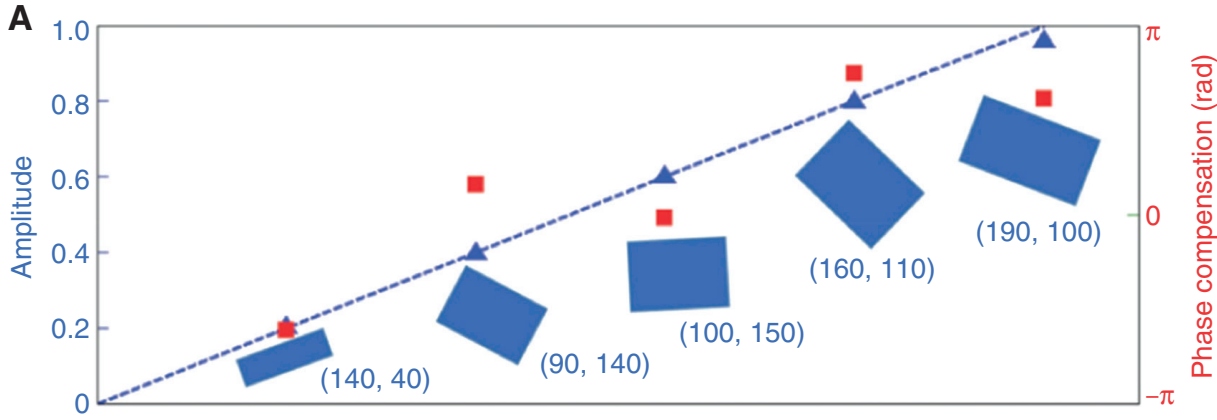

B
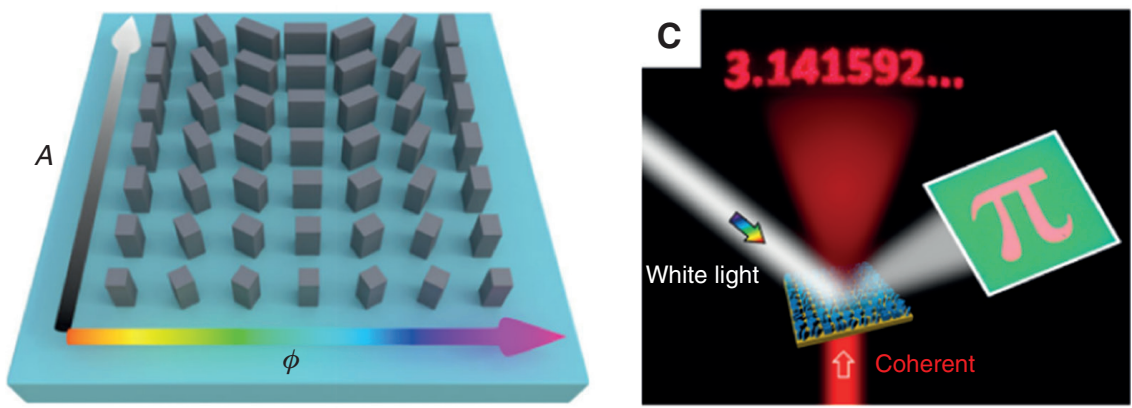

Figure 17: Metasurfaces with three design degrees of freedom for amplitude and phase manipulation.

(A and B) Complex amplitude manipulation enabled with varied dimensions and orientations of nanostructures [129, 130]. (C) Concept of simultaneous nanoprintings and metaholography with spectral and phase manipulations [131]. Reprint permission obtained from [129-131]. 


\subsection{Independent phase control in two polarization directions}

In terms of lightwave manipulation in two orthogonal polarization directions, Arbabi et al. proposed the independent phase manipulation and arbitrary polarization control with a single metasurface [132] simply by changing the dimensions and orientation angles of nanostructures. When a group of LP or CP light with orthogonal polarization states is incident, the metasurface can not only independently control the phase of output light but also arbitrarily control the polarization states of output light (Figure 18A). This scheme, which combines the geometric phase with the propagation phase, can break the fact that the modulated phases by GEMS are equal for LCP and RCP incident light, except for the opposite signs. Using this principle, Grover et al. designed an offaxis metalens that can realize off-axis beam focusing for both LCP and RCP light, and the off-axis amounts can be designed independently (Figure 18B) [133]. Fan et al. designed a metasurface of accelerating light beams with different acceleration directions and caustic trajectories when LCP and RCP light are incident (Figure 18C) [134]. Ding et al. realized a multiplexed metasurface with different orbital angular momenta when CP light with different handedness is incident on the metasurface (Figure 18D) [135]. In addition to the capability of independently controlling the phase of orthogonal LP or CP light, Mueller et al. proved that metasurface with three design degrees of freedom can independently control the phase of any orthogonal polarization light (Figure 18E) [136]. Later, $\mathrm{Wu}$ et al. proved that even if the polarization directions of the two incident light beams are nonorthogonal, the phase can be independently controlled by metasurfaces (Figure 18F) [137].

\subsection{Independent phase control in multiple polarization modes}

With the help of a bulky polarizer and an analyzer, metasurfaces with three design degrees of freedom can be employed to control the phase of multiple polarization modes independently. In 2018, Zhao et al. designed a multichannel metasurface hologram with three polarization modes: $x$-polarization incidence, $x$-polarization decoding; $x$-polarization incidence, $y$-polarization decoding; and $y$-polarization incidence, $y$-polarization decoding (Figure 19A) [138]. Therefore, the designed hologram can produce three independent holographic images. The combination of the three working modes can produce
$2^{3}-1$ diffraction patterns. In 2019, Hu et al. combined the three colors of red, green, and blue with the three working modes and ingeniously realized the noninterleaved and crosstalk-free color metaholography (Figure 19B) [139]. In the same year, Rubin et al. reported their research results of metasurface polarization camera [140]. Using metasurface with three degrees of freedom, the light in four different polarization states from an object is diffracted to different directions (Figure 19C), and the intensity distribution of the object wave in each direction is analyzed, and then the polarization information of the object wave can be obtained. The compact polarization camera is expected to be used in machine vision and remote sensing.

\subsection{Chromatic aberration correction}

The three design degrees of freedom can also be used for the dispersion compensation of metalens by changing the anisotropy and orientation of the nanostructures [141-144]. In 2017, Wang et al. realized the reflective achromatic metalens in a wide band of 1200-1680 nm (Figure 19D) [141]. In 2018, they published a paper on achromatic metalens on Nature Nanotechnology, which ingeniously combined propagation phase and geometric phase to achieve the correction of chromatic aberration in the broad range of visible light (400-660 nm) [143]. Almost at the same time, the Capasso research group from Harvard University realized the functionality of combining propagation phase and geometric phase using coupled phase-shift elements (each element consists of two nanofins in close proximity and can act as a coupled waveguide). As a result, chromatic aberration was corrected in the visible light range (470-670 nm), as shown in Figure 19E. The result is also published in Nature Nanotechnology [142]. Correction of chromatic aberration is expected to solve the most difficult problem in the field of optical imaging.

\section{Conclusions and outlook}

The outstanding advantages of metasurfaces, such as precise lightwave manipulation, ultracompact structures, multifunctional integration, and compatibility with semiconductor process, have been widely accepted by scientists and technicians and have great development prospects. We review the advances from a viewpoint in exploiting the degrees of freedom in the nanostructured metasurface design, designing single and multifunctional optical metasurfaces and their applications. From the 


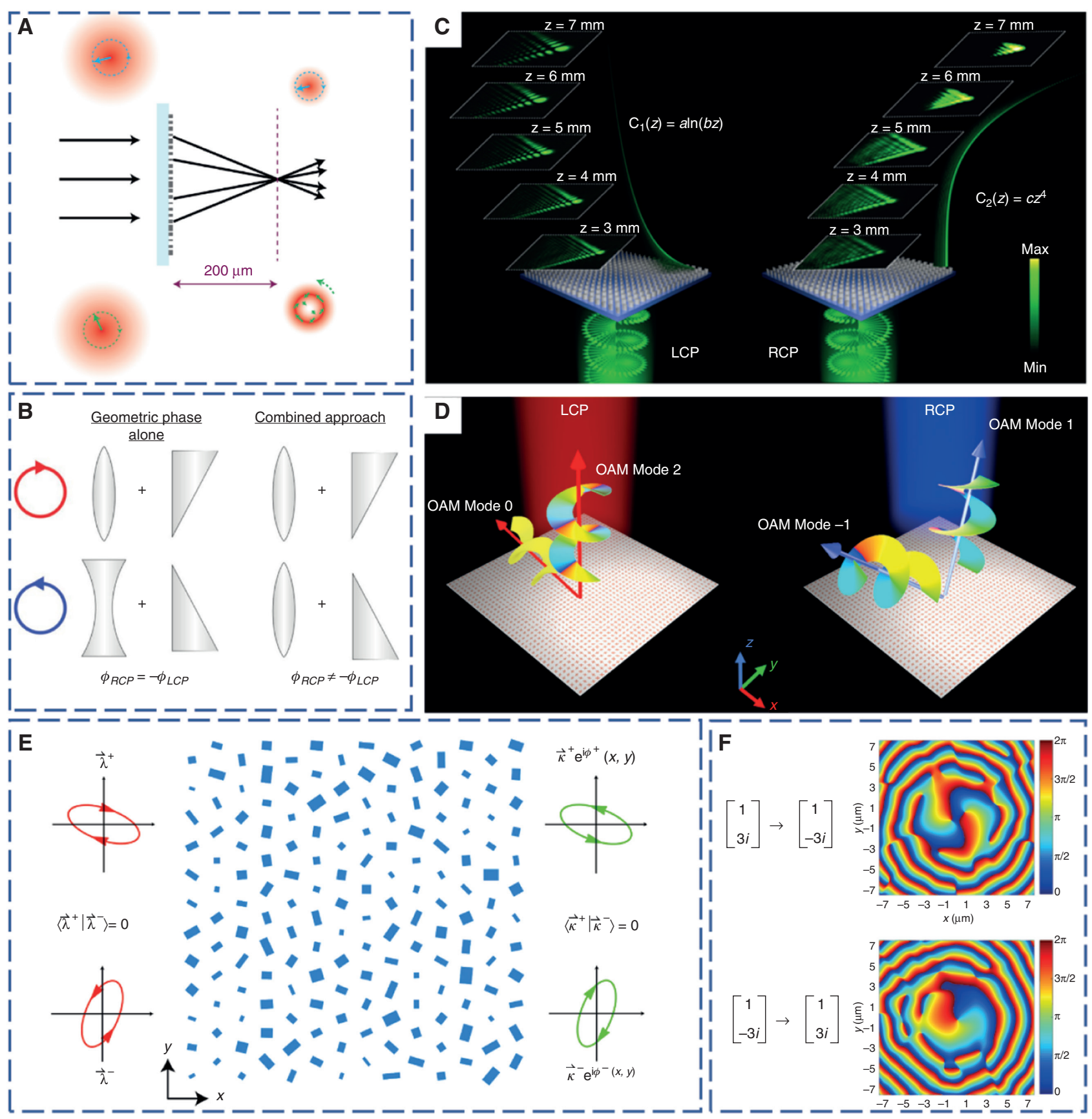

Figure 18: Nanostructured metasurfaces with three design degrees of freedom for phase manipulation in two polarization states. (A) Independent phase manipulation with incidence of two orthogonal polarized light beams [132]. (B) Metasurface design to obtain different off-axis amounts in two orthogonal polarization states [133]. (C) Metasurfaces for accelerating light with different acceleration directions and caustic trajectories [134]. (D) Metasurfaces for generating vortex beams with different topological charges [135]. (E) Independent phase manipulation of metasurfaces in two arbitrary orthogonal polarization states [136]. (F) Independent phase manipulation of metasurfaces in two arbitrary nonorthogonal polarization states [137]. Reprint permission obtained from [132-137].

current trend of metasurface development, the following issues are worthy of further in-depth study.

(1) Stacked metasurfaces. More and more attention has been paid to stacking multiple metasurfaces in the direction of optical axis, which can effectively increase the design degrees of freedom and realize complex functionalities. Avayu et al. [145] and Zhou et al. [146] realized the achromatic lens for red, green, and blue light with three layers of metasurfaces (Figure 20A). Multichannel image displays (Figure 20B and C) [147, 148, 151], nonreciprocal 

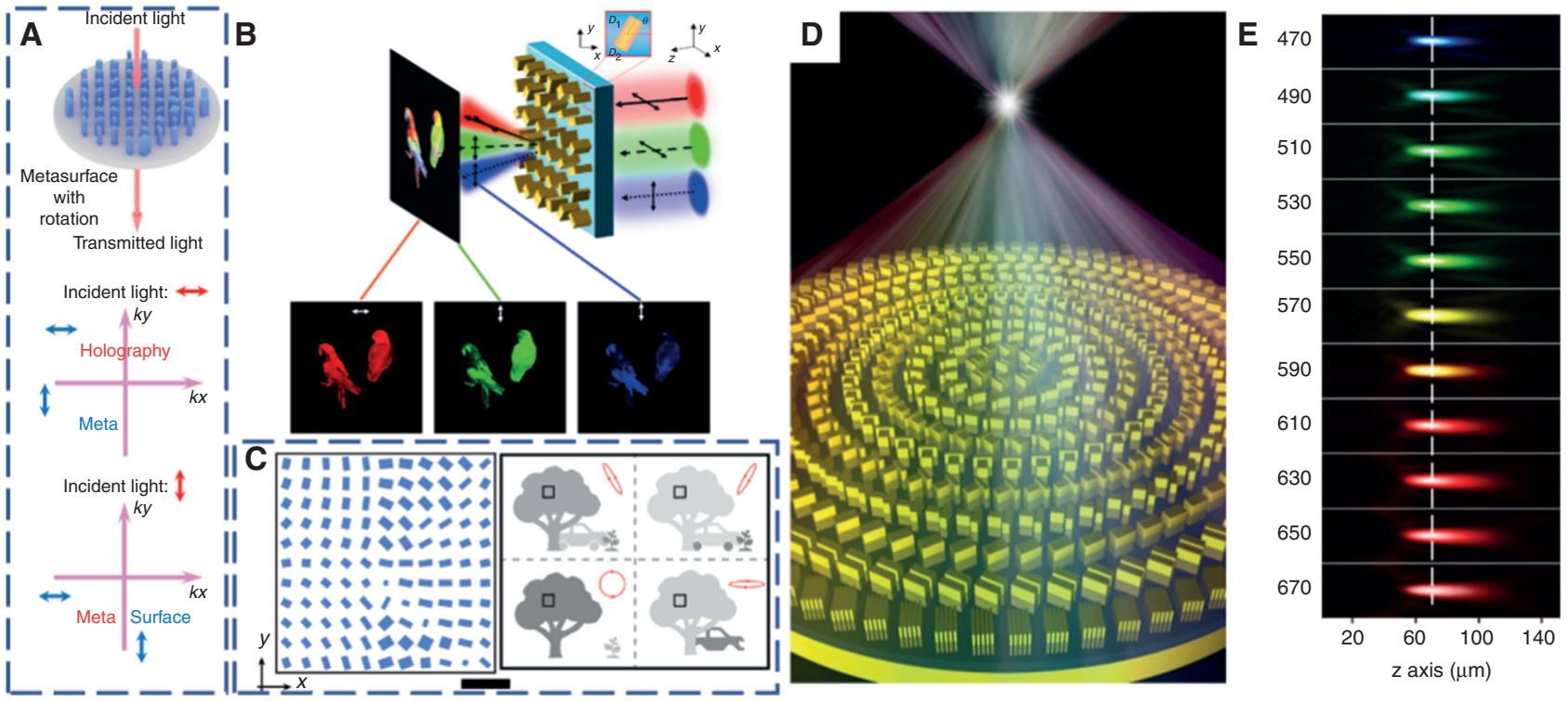

Figure 19: Nanostructures with three design degrees of freedom for phase manipulation in multiple polarization modes.

(A) Multichannel metaholography [138]. (B) Color metaholography [139]. (C) Polarization camera [140]. (D and E) Achromatic metalenses in IR and visible light ranges, respectively [141, 142]. Reprint permission obtained from [138-142].
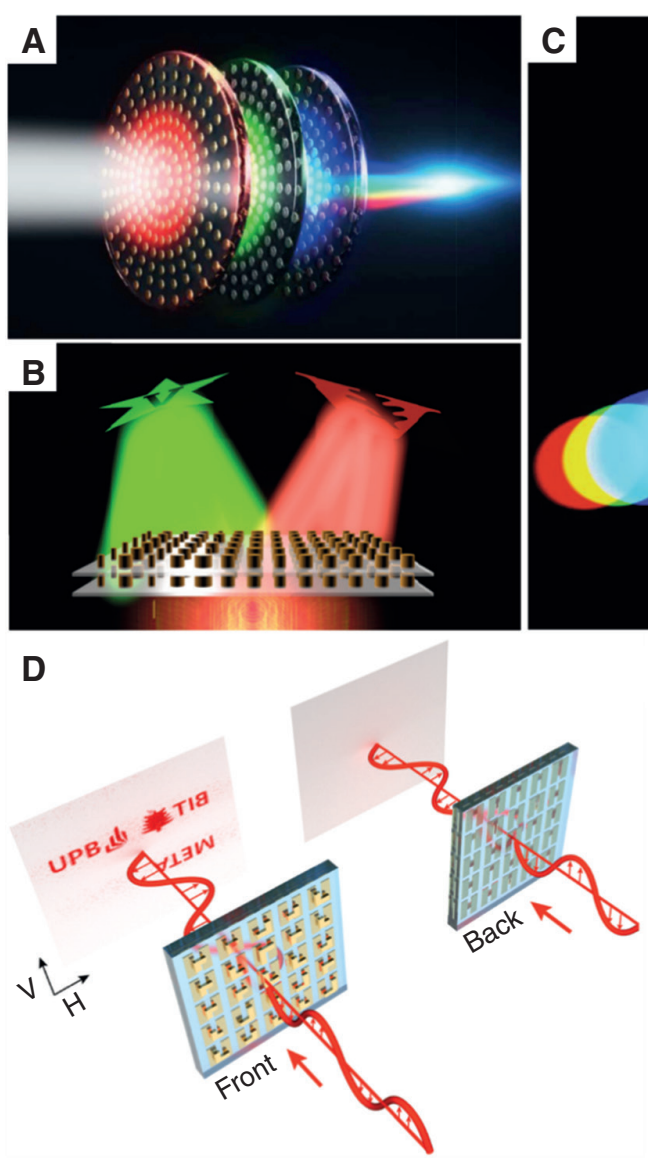

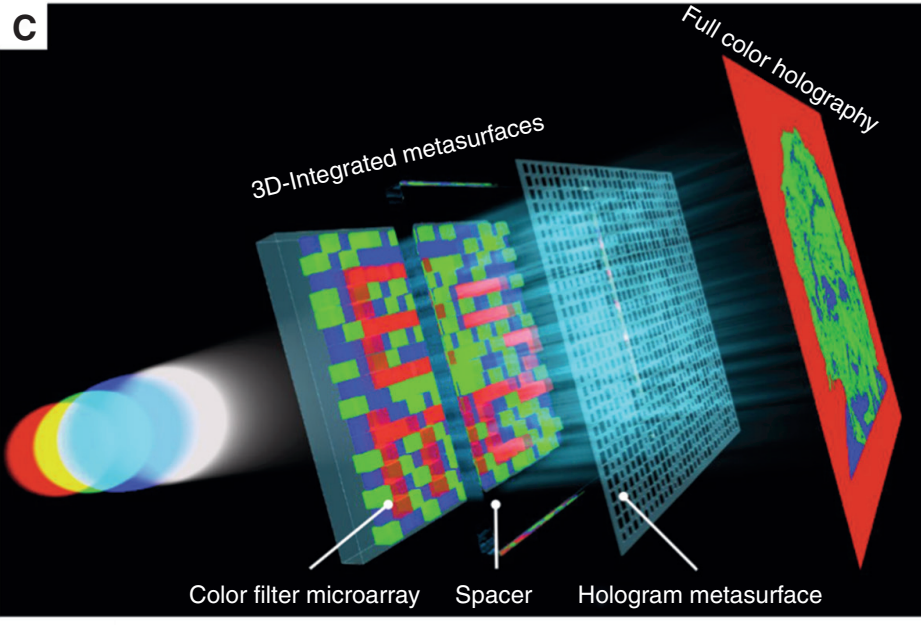

E

Backward



Forward

Figure 20: Stacked metasurfaces.

(A) Achromatic lens for red, green, and blue light [145]. (B and C) Multichannel image displays [147, 148]. (D and E) Nonreciprocal image displays $[149,150]$. Reprint permission obtained from [145, 147-150]. 
image displays (Figure 20D and E) [149, 150], and many other functionalities can also be attained using stacked metasurfaces. By reducing the optical crosstalk between different layers, it is expected to make the stacked metasurfaces achieve more powerful lightwave control ability.

(2) Dynamic metasurfaces. Once metasurfaces are designed and fabricated, their functionality will also be fixed and cannot be changed, thus losing the ability of dynamic manipulation. Realizing the dynamic control of lightwave by metasurfaces has been the expectation of many fields in applied optics. In a sense, dynamic control is also a new design degree of freedom (namely, time division multiplexing [6]). Recently, multifunctional switching can be realized using phase-changed materials (Figure 21A) [152, 155], stretchable flexible substrates (Figure 21B) [153, 156], and reconfigurable chemical methods (Figure 21C) [154, 157-159], but there are also some shortcomings such as complex nanostructures, design difficulty, time consumption, and limited adjustment range. The combination of metasurface with electricity, heat, and magnetism can realize the electronic, temperature, and magnetic control of metasurface devices with high spatial and time resolution, which is expected to achieve more flexible control of lightwave. The dynamic metasurface devices, which are small in size, light in weight, low in power consumption, and easy to be integrated, have very important application prospects in lidar, dynamic display, and adaptive optics.

(3) High-performance achromatic metalens. Highperformance metalens for optical imaging and sensing can meet the eagerness of consumer optoelectronics toward being lightweight, ultracompact, flexible, and wearable. Recently, although there have been ingenious schemes for achromatic aberration correction, metalenses usually have a moderate and unbalanced optical transmission efficiencies for different wavelengths compared to conventional achromatic lens. In addition, to meet the commercial requirements, some other issues for both lens design and fabrication, such as NA, FOVs, vignetting, optical zooming, and large-scale and mass manufacturing, should be considered seriously.

Acknowledgments: This project was supported by the National Natural Science Foundation of China (grants 91950110, 11774273, 11904267, Funder Id: http://dx.doi. org/10.13039/501100001809), Postdoctoral Innovation Talent Support Program of China (grant BX20180221), China Postdoctoral Science Foundation (grant 2019M652688, Funder Id: http://dx.doi.org/10.13039/501100002858), and Natural Science Foundation of Jiangsu Province (grant BK20190211).
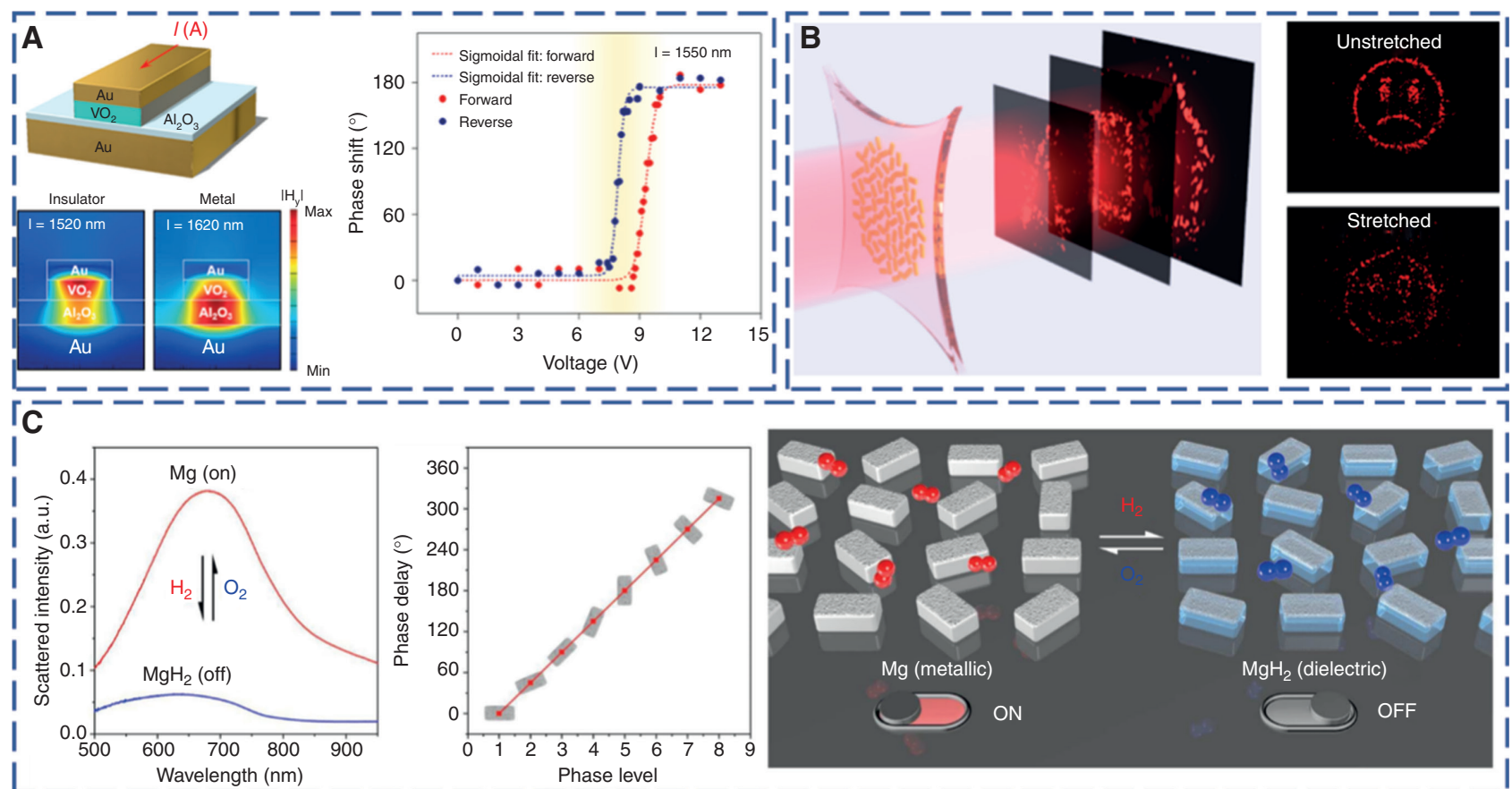

Figure 21: Dynamic metasurfaces using (A) phase-changed materials [152], (B) stretchable flexible substrates [153], and (C) reconfigurable chemical methods [154].

Reprint permission obtained from [152-154]. 


\section{References}

[1] Kamali SM, Arbabi E, Arbabi A, Faraon A. A review of dielectric optical metasurfaces for wavefront control. Nanophotonics 2018;7:1041-68.

[2] Jeong H, Yang $\mathrm{Y}$, Cho H, et al. Emerging advanced metasurfaces: alternatives to conventional bulk optical devices. Microelectron Eng 2020;220:111146.

[3] Luo X, Tsai D, Gu M, Hong M. Extraordinary optical fields in nanostructures: from sub-diffraction-limited optics to sensing and energy conversion. Chem Soc Rev 2019;48:2458-94.

[4] Wen D, Yue F, Liu W, Chen S, Chen X. Geometric metasurfaces for ultrathin optical devices. Adv Opt Mater 2018;6:1800348.

[5] Neshev D, Aharonovich I. Optical metasurfaces: new generation building blocks for multi-functional optics. Light Sci Appl 2018;7:58.

[6] Chen S, Liu W, Li Z, Cheng H, Tian J. Metasurface-empowered optical multiplexing and multifunction. Adv Mater 2020;32:1805912.

[7] Bao L, Cui TJ. Tunable, reconfigurable, and programmable metamaterials. Microw Opt Technol Lett 2020;62:9-32.

[8] Deng ZL, Li G. Metasurface optical holography. Mater Today Phys 2017;3:16-32.

[9] Jiang Q, Jin G, Cao L. When metasurface meets hologram: principle and advances. Adv Opt Photonics 2019;11:518-76.

[10] Wan W, Gao J, Yang X. Metasurface holograms for holographic imaging. Adv Opt Mater 2017;5:1700541.

[11] Tseng ML, Hsiao HH, Chu CH, et al. Metalenses: advances and applications. Adv Opt Mater 2018;6:1800554.

[12] Yu N, Genevet P, Kats MA, et al. Light propagation with phase discontinuities: generalized laws of reflection and refraction. Science 2011;334:333-7.

[13] Aieta F, Genevet P, Kats MA, et al. Aberration-free ultrathin flat lenses and axicons at telecom wavelengths based on plasmonic metasurfaces. Nano Lett 2012;12:4932-6.

[14] Qin F, Ding L, Zhang L, et al. Hybrid bilayer plasmonic metasurface efficiently manipulates visible light. Sci Adv 2016;2:e1501168.

[15] Ni X, Kildishev AV, Shalaev VM. Metasurface holograms for visible light. Nat Commun 2013;4:2807.

[16] Goebel B, Wang LL, Tschudi T. Multilayer technology for diffractive optical elements. Appl Opt 1996;35:4490-3.

[17] Zarschizky H, Stemmer A, Mayerhofe F, Lefranc G, Gramann W. Binary and multilevel diffractive lenses with submicrometer feature sizes. Opt Eng 1994;33:3527-36.

[18] Xie T, Guo S, Chen Z. GRIN lens rod based probe for endoscopic spectral domain optical coherence tomography with fast dynamic focus tracking. Opt Express 2006;14:3238-46.

[19] Ye C, McLeod RR. GRIN lens and lens array fabrication with diffusion-driven photopolymer. Opt Lett 2008;33:2575-7.

[20] Paul O, Reinhard B, Krolla B, Beigang R, Rahm M. Gradient index metamaterial based on slot elements. Appl Phys Lett 2010;96:241110.

[21] Verslegers L, Catrysse PB, Yu Z, Shin W, Ruan Z, Fan S. Phase front design with metallic pillar arrays. Opt Lett 2010;35:844-6.

[22] Arbabi A, Horie Y, Ball AJ, Bagheri M, Faraon A. Subwavelengththick lenses with high numerical apertures and large efficiency based on high-contrast transmitarrays. Nat Commun 2015;6:7069.
[23] Chong KE, Staude I, James A, et al. Polarization-independent silicon metadevices for efficient optical wavefront control. Nano Lett 2015;15:5369-74.

[24] Li QT, Dong F, Wang B, et al. Polarization-independent and high-efficiency dielectric metasurfaces for visible light. Opt Express 2016;24:16309-19.

[25] Fan ZB, Shao ZK, Xie MY, et al. Silicon nitride metalenses for close-to-one numerical aperture and wide-angle visible imaging. Phys Rev Appl 2017;10:014005.

[26] Khorasaninejad M, Zhu AY, Roques-Carmes C, et al. Polarization-insensitive metalenses at visible wavelengths. Nano Lett 2016;16:7229-34.

[27] Zhang Y, Mittra R, Hong W. On the synthesis of a flat lens using a wideband low-reflection gradient-index metamaterial. J Electromagnet Wave 2011;25:2178-87.

[28] Zhan A, Colburn S, Trivedi R, Fryett TK, Dodson CM, Majumdar A. Low-contrast dielectric metasurface optics. ACS Photonics 2016;3:209-14.

[29] Arbabi A, Briggs RM, Horie Y, Bagheri M, Faraon A. Efficient dielectric metasurface collimating lenses for mid-infrared quantum cascade lasers. Opt Express 2015;23:33310-7.

[30] Arbabi A, Arbabi E, Horie Y, Kamali SM, Han S, Faraon A. Aberration corrected metasurface doublet lens. CLEO 2016:STh1E.6.

[31] Arbabi A, Arbabi E, Horie Y, Kamali SM, Faraon A. Planar metasurface retroreflector. Nat Photonics 2017;11:415-20.

[32] Colburn S, Zhan A, Majumdar A. Varifocal zoom imaging with large area focal length adjustable metalenses. Optica 2018;5:825-31.

[33] Pahlevaninezhad H, Khorasaninejad M, Huang YW, et al. Nano-optic endoscope for high-resolution optical coherence tomography in vivo. Nat Photonics 2018;12:540-7.

[34] Shrestha S, Overvig AC, Lu M, Stein A, Yu N. Broadband achromatic dielectric metalenses. Light Sci Appl 2018;7:85.

[35] Huang K, Liu H, Garcia-Vidal F], et al. Ultrahigh-capacity nonperiodic photon sieves operating in visible light. Nat Commun 2015;6:7059.

[36] Huang K, Liu H, Si G, Wang Q, Lin J, Teng J. Photon-nanosieve for ultrabroadband and large-angle-of-view holograms. Laser Photonics Rev 2017;11:1700025.

[37] Xu Z, Huang L, Li X, Tang C, Wei Q, Wang Y. Quantitatively correlated amplitude holography based on photon sieves. Adv Opt Mater 2020;8:1901169.

[38] Tan SJ, Zhang L, Zhu D, et al. Plasmonic color palettes for photorealistic printing with aluminum nanostructures. Nano Lett 2014;14:4023-9.

[39] Dai P, Wang Y, Zhu X, et al. Transmissive structural color filters using vertically coupled aluminum nanohole/nanodisk array with a triangular-lattice. Nanotechnology 2018;29:395202.

[40] Li Z, Palacios E, Butun S, Aydin K. Visible-frequency metasurfaces for broadband anomalous reflection and high-efficiency spectrum splitting. Nano Lett 2015;15:1615-21.

[41] Li Z, Palacios E, Butun S, Aydin K. Ultrawide angle, directional spectrum splitting with visible-frequency versatile metasurfaces. Adv Opt Mater 2016;4:953-8.

[42] Yang L, Wu D, Liu Y, et al. High-efficiency all-dielectric transmission metasurface for linearly polarized light in the visible region. Photonics Res 2018;6:517-24.

[43] Sun S, Yang KY, Wang CM, et al. High-efficiency broadband anomalous reflection by gradient meta-surfaces. Nano Lett 2012;12:6223-9. 
[44] Shalaev MI, Sun J, Tsukernik A, Pandey A, Nikolskiy K, Litchinitser NM. High-efficiency all-dielectric metasurfaces for ultracompact beam manipulation in transmission mode. Nano Lett 2015;15:6261-6.

[45] Andreas T, Aleksandrs L, Mingkai L, et al. Imaging-based molecular barcoding with pixelated dielectric metasurfaces. Science 2018;360:1105-9.

[46] Yang Y, Wang W, Moitra P, Kravchenko II, Briggs DP, Valentine J. Dielectric meta-reflectarray for broadband linear polarization conversion and optical vortex generation. Nano Lett 2014;14:1394-9.

[47] Wang W, Guo Z, Zhou K, et al. Polarization-independent longitudinal multi-focusing metalens. Opt Express 2015;23:29855-66.

[48] Wang Q, Xu Q, Zhang X, et al. All-dielectric meta-holograms with holographic images transforming longitudinally. ACS Photonics 2018;5:599-606.

[49] Gao S, Yue W, Park CS, Lee SS, Kim ES, Choi DY. Aluminum plasmonic metasurface enabling a wavelength-insensitive phase gradient for linearly polarized visible light. ACS Photonics 2017;4:322-8.

[50] Pors A, Nielsen MG, Eriksen RL, Bozhevolnyi SI. Broadband focusing flat mirrors based on plasmonic gradient metasurfaces. Nano Lett 2013;13:829-34.

[51] Yesilkoy M, Arvelo ER, Jahani Y, et al. Ultrasensitive hyperspectral imaging and biodetection enabled by dielectric metasurfaces. Nat Photonics 2019;13:390-6.

[52] Kruk S, Hopkins B, Kravchenko II, Miroshnichenko A, Neshev DN, Kivshar YS. Broadband highly efficient dielectric metadevices for polarization control. APL Photonics 2016;1:030801.

[53] Zhong J, An N, Yi N, Zhu M, Song Q, Xiao S. Broadband and tunable-focus flat lens with dielectric metasurface. Plasmonics 2016;11:537-41.

[54] Ding F, Wang Z, He S, Shalaev VM, Kildishev AV. Broadband high-efficiency half-wave plate: a supercell-based plasmonic metasurface approach. ACS Nano 2015;9:4111-9.

[55] Deng J, Li Z, Zheng G, et al. Depth perception based 3D holograms enabled with polarization-independent metasurfaces. Opt Express 2018;26:11843-9.

[56] Fu R, Li Z, Zheng G, et al. Reconfigurable step-zoom metalens without optical and mechanical compensations. Opt Express 2019;27:12221-30.

[57] Yang B, Liu W, Li Z, Cheng H, Chen S, Tian J. Polarization-sensitive structural colors with hue-and-saturation tuning based on all-dielectric nanopixels. Adv Opt Mater 2018;6:1701009.

[58] Jang J, Jeong H, Hu G, Qiu CW, Nam KT, Rho J. Kerker-conditioned dynamic cryptographic nanoprints. Adv Opt Mater 2019;7:1801070.

[59] Goh XM, Zheng Y, Tan SJ, et al. Three-dimensional plasmonic stereoscopic prints in full colour. Nat Commun 2014;5:5361.

[60] Zhang Y, Shi L, Hu D, et al. Full-visible multifunctional aluminium metasurfaces by in situ anisotropic thermoplasmonic laser printing. Nanoscale Horiz 2019;4:601-9.

[61] Martins A, Li J, Mota AFD, et al. Broadband c-Si metasurfaces with polarization control at visible wavelengths: applications to 3D stereoscopic holography. Opt Express 2018;26:30740-52.

[62] Xie Z, Lei T, Si G, et al. Meta-holograms with full parameter control of wavefront over a $1000 \mathrm{~nm}$ bandwidth. ACS Photonics 2017;4:2158-64.
[63] Bomzon Z, Biener G, Kleiner V, Hasman E. Space-variant Pancharatnam-Berry phase optical elements with computergenerated subwavelength gratings. Opt Lett 2002;27:1141-3.

[64] Levy U, Tsai CH, Kim HC, Fainman Y. Design, fabrication and characterization of subwavelength computer-generated holograms for spot array generation. Opt Express 2004;12:5345-55.

[65] Levy U, Kim HC, Tsai CH, Fainman Y. Near-infrared demonstration of computer-generated holograms implemented by using subwavelength gratings with space-variant orientation. Opt Lett 2005;30:2089-91.

[66] Huang L, Chen X, Mühlenbernd H, et al. Dispersionless phase discontinuities for controlling light propagation. Nano Lett 2012;12:5750-5.

[67] Huang L, Chen X, Mühlenbernd H, et al. Three-dimensional optical holography using a plasmonic metasurface. Nat Commun 2013;4:2808.

[68] Gao H, Pu M, Li X, et al. Super-resolution imaging with a Bessel lens realized by a geometric metasurface. Opt Express 2017;25:13933-43.

[69] Zheng G, Mühlenbernd H, Kenney M, Li G, Zentgraf T, Zhang S. Metasurface holograms reaching $80 \%$ efficiency. Nat Nanotechnol 2015;10:308-12.

[70] Khorasaninejad M, Crozier KB. Silicon nanofin grating as a miniature chirality-distinguishing beam-splitter. Nat Commun 2014;5:5386.

[71] Li Z, Zheng G, He P, et al. All-silicon nanorod-based Dammann gratings. Opt Lett 2015;40:4285-8.

[72] Khorasaninejad M, Chen WT, Devlin RC, Oh J, Zhu AY, Capasso F. Metalenses at visible wavelengths: diffraction-limited focusing and subwavelength resolution imaging. Science 2016;352:1190-4.

[73] Li Z, Kim I, Zhang L, et al. Dielectric meta-holograms enabled with dual magnetic resonances in visible light. ACS Nano 2017;11:9382-9.

[74] Li Z, Dai Q, Mehmood M Q, et al. Full-space cloud of random points with a scrambling metasurface. Light Sci Appl 2018;7:63.

[75] Lin D, Fan P, Hasman E, Brongersma ML. Dielectric gradient metasurface optical elements. Science 2014;345:298-302.

[76] Devlin RC, Khorasaninejad M, Chen WT, Oh J, Capasso F. Broadband high-efficiency dielectric metasurfaces for the visible spectrum. Proc Natl Acad Sci 2016;113:10473-8.

[77] Groever B, Chen WT, Capasso F. Meta-lens doublet in the visible region. Nano Lett 2017;17:4902-7.

[78] Zheng G, Liu G, Kenney MG, et al. Ultracompact high-efficiency polarising beam splitter based on silicon nanobrick arrays. Opt Express 2016;24:6749-57.

[79] Ellenbogen T, Seo K, Crozier KB. Chromatic plasmonic polarizers for active visible color filtering and polarimetry. Nano Lett 2012;12:1026-31.

[80] Yue F, Zhang C, Zang XF, et al. High-resolution grayscale image hidden in a laser beam. Light Sci Appl 2018;7:17129.

[81] Dai Q, Deng L, Deng J, et al. Ultracompact, high-resolution and continuous grayscale image display based on resonant dielectric metasurfaces. Opt Express 2019;27:27927-35.

[82] Deng J, Deng L, Guan Z, et al. Multiplexed anticounterfeiting meta-image displays with single-sized nanostructures. Nano Lett 2020;20:1830-8.

[83] Chen X, Huang L, Mühlenbernd H, et al. Dual-polarity plasmonic metalens for visible light. Nat Commun 2012;3:1198. 
[84] Zheng G, Wu W, Li Z, et al. Dual field-of-view step-zoom metalens. Opt Lett 2017;42:1261-4.

[85] Cui Y, Zheng G, Chen M, et al. Reconfigurable continuouszoom metalens in visible band. Chin Opt Lett 2019;17:111603.

[86] Chen X, Chen M, Mehmood MQ, et al. Longitudinal multifoci metalens for circularly polarized light. Adv Opt Mater 2015;3:1201-6.

[87] Mehmood MQ, Mei S, Hussain S, et al. Visible-frequency metasurface for structuring and spatially multiplexing optical vortices. Adv Mater 2016;28:2533-9.

[88] Wen D, Yue F, Ardron M, Chen X. Multifunctional metasurface lens for imaging and Fourier transform. Sci Rep 2016;6:27628.

[89] Khorasaninejad M, Chen WT, Zhu AY, et al. Visible wavelength planar metalenses based on titanium dioxide. IEEE J Quantum Elect 2017;23:43-58.

[90] Wen D, Yue F, Li G, et al. Helicity multiplexed broadband metasurface holograms. Nat Commun 2015;6:8241.

[91] Huang K, Deng J, Leong HS, et al. Ultraviolet metasurfaces of $\approx 80 \%$ efficiency with antiferromagnetic resonances for optical vectorial anti-counterfeiting. Laser Photonics Rev 2019;13:1800289.

[92] Zhang X, Yang S, Yue W, et al. Direct polarization measurement using a multiplexed Pancharatnam-Berry metahologram. Optica 2019;6:1190-8.

[93] Wang Q, Plum E, Yang Q, et al. Reflective chiral meta-holography: multiplexing holograms for circularly polarized waves. Light Sci Appl 2018;7:25.

[94] Zhang C, Dong F, Intaravanne Y, et al. Multichannel metasurfaces for anticounterfeiting. Phys Rev Appl 2019;12:034028.

[95] Zhao W, Liu B, Jiang H, Song J, Pei Y, Jiang Y. Full-color hologram using spatial multiplexing of dielectric metasurface. Opt Lett 2016;41:147-50.

[96] Wang B, Dong F, Li QT, et al. Visible-frequency dielectric metasurfaces for multiwavelength achromatic and highly dispersive holograms. Nano Lett 2016;16:5235-40.

[97] Wang B, Dong F, Yang D, et al. Polarization-controlled colortunable holograms with dielectric metasurfaces. Optica 2017;4:1368-71.

[98] Zang X, Dong F, Yue F, et al. Polarization encoded color image embedded in a dielectric metasurface. Adv Mater 2018;30:1707499.

[99] Wei Q, Sain B, Wang Y, et al. Simultaneous spectral and spatial modulation for color printing and holography using all-dielectric metasurfaces. Nano Lett 2019;19:8964-71.

[100] Zhang X, Jin J, Pu M, et al. Ultrahigh-capacity dynamic holographic displays via anisotropic nanoholes. Nanoscale 2017;9:1409-15.

[101] Li X, Chen L, Li Y, et al. Multicolor 3D meta-holography by broadband plasmonic modulation. Sci Adv 2016;2:e1601102.

[102] Zhang X, Pu M, Guo Y, et al. Colorful metahologram with independently controlled images in transmission and reflection spaces. Adv Funct Mater 2019;29:1809145.

[103] Jin L, Huang YW, Jin Z, et al. Dielectric multi-momentum metatransformer in the visible. Nat Commun 2019;10:4789.

[104] Wan W, Gao J, Yang X. Full-color plasmonic metasurface holograms. ACS Nano 2016;10:10671-80.

[105] Jin L, Dong Z, Mei S, et al. Noninterleaved metasurface for ( $\left.2^{6}-1\right)$ spin- and wavelength-encoded holograms. Nano Lett 2018;18:8016-24.

[106] Ye W, Zeuner F, Li X, et al. Spin and wavelength multiplexed nonlinear metasurface holography. Nat Commun 2016;7:11930.
[107] Huang K, Dong Z, Mei S, et al. Silicon multi-meta-holograms for the broadband visible light. Laser Photonics Rev 2016;10:500-9.

[108] Wei Q, Huang L, Li X, Liu J, Wang Y. Broadband multiplane holography based on plasmonic metasurface. Adv Opt Mater 2017;5:1700434.

[109] Deng J, Yang Y, Tao J, et al. Spatial frequency multiplexed meta-holography and meta-nanoprinting. ACS Nano 2019;13:9237-46.

[110] Lee G, Yoon G, Lee SY, et al. Complete amplitude and phase control of light using broadband holographic metasurfaces. Nanoscale 2018;10:4237-45.

[111] Deng ZL, Deng J, Zhuang X, et al. Diatomic metasurface for vectorial holography. Nano Lett 2018;18:2885-92.

[112] Wang S, Li F, Deng J, et al. Diatomic metasurface based broadband J-plate for arbitrary spin-to-orbital conversion. J Phys D Appl Phys 2019;52:324002.

[113] Deng ZL, Deng J, Zhuang X, et al. Facile metagrating holograms with broadband and extreme angle tolerance. Light Sci Appl 2018;7:78.

[114] Deng ZL, Jin M, Ye X, et al. Full-color complex-amplitude vectorial holograms based on multi-freedom metasurfaces. Adv Funct Mater 2020;30:1910610.

[115] Bao Y, Yu Y, Xu H, et al. Full-colour nanoprint-hologram synchronous metasurface with arbitrary hue-saturation brightness control. Light Sci Appl 2019;8:95.

[116] Bao Y, Ni J, Qiu CW. A minimalist single-layer metasurface for arbitrary and full control of vector vortex beams. Adv Mater 2020;32:1905659.

[117] Khorasaninejad M, Chen WT, Oh J, Capasso F. Super-dispersive off-axis meta-lenses for compact high resolution spectroscopy. Nano Lett 2016;16:3732-7.

[118] Yang Z, Wang Z, Wang Y, et al. Generalized Hartmann-Shack array of dielectric metalens sub-arrays for polarimetric beam profiling. Nat Commun 2018;9:4607.

[119] Yuan G, Rogers ETF, Zheludev NI. "Plasmonics" in free space: observation of giant wavevectors, vortices, and energy backflow in superoscillatory optical fields. Light Sci Appl 2019;8:2.

[120] Yuan GH, Zheludev NI. Detecting nanometric displacements with optical ruler metrology. Science 2019;364:771-5.

[121] Zhou J, Qian H, Chen CF, et al. Optical edge detection based on high-efficiency dielectric metasurface. Proc Natl Acad Sci 2019;116:11137-40.

[122] Liu HC, Yang B, Guo Q, et al. Single-pixel computational ghost imaging with helicity-dependent metasurface hologram. Sci Adv 2017;3:e1701477.

[123] Yue F, Zang X, Wen D, et al. Geometric phase generated optical illusion. Sci Rep 2017;7:11440.

[124] Lee GY, Hong JY, Hwang S, et al. Metasurface eyepiece for augmented reality. Nat Commun 2018;9:4562.

[125] Xu HX, Ma S, Ling X, et al. Deterministic approach to achieve broadband polarization-independent diffusive scatterings based on metasurfaces. ACS Photonics 2018;5:1691-702.

[126] Liu X, Deng J, Li KF, et al. Optical metasurfaces for designing planar Cassegrain-Schwarzschild objectives. Phys Rev Appl 2019;11:054055.

[127] Chen C, Song W, Chen J, et al. Spectral tomographic imaging with aplanatic metalens. Light Sci Appl 2019;8:99.

[128] Yu H, Jiang M, Guo Y, et al. Plasmonic metasurfaces with high UV-vis transmittance for photopatterning of designer molecu lar orientations. Adv Opt Mater 2019;7:1900117. 
[129] Song X, Huang L, Tang C, et al. Selective diffraction with complex amplitude modulation by dielectric metasurfaces. Adv Opt Mater 2018;6:1701181.

[130] Overvig AC, Shrestha S, Malek SC, et al. Dielectric metasurfaces for complete and independent control of the optical amplitude and phase. Light Sci Appl 2019;8:92.

[131] Yoon G, Lee D, Nam KT, Rho J. “Crypto-display” in dual-mode metasurfaces by simultaneous control of phase and spectral responses. ACS Nano 2018;12:6421-8.

[132] Arbabi A, Horie Y, Bagheri M, Faraon A. Dielectric metasurfaces for complete control of phase and polarization with subwavelength spatial resolution and high transmission. Nat Nanotechnol 2015;10:937-43.

[133] Groever B, Rubin NA, Mueller JPB, Devlin RC, Capasso F. Highefficiency chiral meta-lens. Sci Rep 2018;8:7240.

[134] Fan Q, Zhu W, Liang Y, et al. Broadband generation of photonic spin-controlled arbitrary accelerating light beams in the visible. Nano Lett 2019;19:1158-65.

[135] Ding G, Chen K, Luo X, Zhao J, Jiang T, Feng Y. Dual-helicity decoupled coding metasurface for independent spin-to-orbital angular momentum conversion. Phys Rev Appl 2019;11:044043.

[136] Mueller JPB, Rubin NA, Devlin RC, Groever B, Capasso F. Metasurface polarization optics: independent phase control of arbitrary orthogonal states of polarization. Phys Rev Lett 2017;118:113901.

[137] Wu L, Tao J, Zheng G. Controlling phase of arbitrary polarizations using both the geometric phase and the propagation phase. Phys Rev B 2018;97:245426.

[138] Zhao R, Sain B, Wei Q, et al. Multichannel vectorial holographic display and encryption. Light Sci Appl 2018;7:95

[139] Hu Y, Li L, Wang Y, et al. Trichromatic and tripolarizationchannel holography with noninterleaved dielectric metasurface. Nano Lett 2020;20:994-1002.

[140] Rubin N, D’Aversa G, Chevalier P, Shi Z, Chen WT, Capasso F. Matrix Fourier optics enables a compact full-Stokes polarization camera. Science 2019;365:eaax1839.

[141] Wang S, Wu PC, Su VC, et al. Broadband achromatic optical metasurface devices. Nat Commun 2017;8:187.

[142] Chen WT, Zhu AY, Sanjeev V, et al. A broadband achromatic metalens for focusing and imaging in the visible. Nat Nanotechnol 2018;13:220-6.

[143] Wang S, Wu PC, Su VC, et al. A broadband achromatic metalens in the visible. Nat Nanotechnol 2018;13:227-32.
[144] Lin RJ, Su VC, Wang S, et al. Achromatic metalens array for full-colour light-field imaging. Nat Nanotechnol 2019;14:227-31.

[145] Avayu O, Almeida E, Prior Y, Ellenbogen T. Composite functional metasurfaces for multispectral achromatic optics. Nat Commun 2017;8:14992.

[146] Zhou Y, Kravchenko II, Wang H, Nolen JR, Gu G, Valentine J. Multilayer noninteracting dielectric metasurfaces for multiwavelength metaoptics. Nano Lett 2018;18:7529-37.

[147] Zhou Y, Kravchenko II, Wang H, Zheng H, Gu G, Valentine J. Multifunctional metaoptics based on bilayer metasurfaces. Light Sci Appl 2019;8:80.

[148] Hu Y, Luo X, Chen Y, et al. 3D-integrated metasurfaces for fullcolour holography. Light Sci Appl 2019;8:86.

[149] Frese D, Wei Q, Wang Y, Huang L, Zentgraf T. Nonreciprocal asymmetric polarization encryption by layered plasmonic metasurfaces. Nano Lett 2019;19:3976-80.

[150] Chen K, Ding G, Hu G, et al. Directional Janus metasurface. Adv Mater 2020;32:1906352.

[151] Luo X, Hu Y, Li X, et al. Integrated metasurfaces with microprints and helicity-multiplexed holograms for real-time optical encryption. Adv Opt Mater 2020;8:1902020.

[152] Kim Y, Wu PC, Sokhoyan R, et al. Phase modulation with electrically tunable vanadium dioxide phase-change metasurfaces. Nano Lett 2019;19:3961-8.

[153] Malek SC, Ee HS, Agarwal R. Strain multiplexed metasurface holograms on a stretchable substrate. Nano Lett 2017;17:3641-5.

[154] Li J, Kamin S, Zheng G, Neubrech F, Zhang S, Liu N. Addressable metasurfaces for dynamic holography and optical information encryption. Sci Adv 2018;4:eaar6768.

[155] Wang D, Zhang L, Gu Y, et al. Switchable ultrathin quarterwave plate in terahertz using active phase-change metasurface. Sci Rep 2015;5:15020.

[156] Chen W, Liu W, Jiang Y, et al. Ultrasensitive, mechanically responsive optical metasurfaces via strain amplification. ACS Nano 2018;12:10683-92.

[157] Yu P, Li J, Li X, et al. Generation of switchable singular beams with dynamic metasurfaces. ACS Nano 2019;13:7100-6.

[158] Duan X, Liu N. Magnesium for dynamic nanoplasmonics. Acc Chem Res 2019;52:1979-89.

[159] Chen Y, Duan X, Matuschek M, et al. Dynamic color displays using stepwise cavity resonators. Nano Lett 2017;17:5555-60. 